\title{
Note on off-shell relations in nonlinear sigma model
}

\author{
Gang Chen, ${ }^{a}$ Yi-Jian Du, ${ }^{b, 1,2}$ Shuyi $\mathbf{L i}^{a}$ and Hanqing Liu ${ }^{a}$ \\ ${ }^{a}$ Department of Physics, Nanjing University, \\ 22 Hankou Road, Nanjing 210093, China \\ ${ }^{b}$ Department of Astronomy and Theoretical Physics, Lund University, \\ Sölvegatan 14A, 22362 Lund, Sweden \\ E-mail: gang.chern@gmail.com, yijian.du@thep.lu.se, \\ shuyili19921123@hotmail.com, physics@hqliu.com
}

ABSTRACT: In this note, we investigate relations between tree-level off-shell currents in nonlinear sigma model. Under Cayley parametrization, all odd-point currents vanish. We propose and prove a generalized U(1) identity for even-point currents. The off-shell U(1) identity given in [1] is a special case of the generalized identity studied in this note. The on-shell limit of this identity is equivalent with the on-shell KK relation. Thus this relation provides the full off-shell correspondence of tree-level KK relation in nonlinear sigma model.

KeYwords: Scattering Amplitudes, Sigma Models

ARXIV EPRINT: 1412.3722

\footnotetext{
${ }^{1}$ Corresponding author.

${ }^{2}$ On leave from Center for Field Theory and Particle Physics, Department of Physics, Fudan University, China.
} 


\section{Contents}

1 Introduction 1

2 Preparation: Feynman rules, Berends-Giele recursion and U(1) identity 3

2.1 Feynman rules 3

2.2 Berends-Giele recursion 4

2.3 The off-shell versions of U(1) identity 4

3 Direct calculation of an eight-point example 5

4 Proof of the generalized U(1) identity for off-shell currents $\quad 7$

4.1 Proof of the validity of (4.2) with an undetermined coefficient $\mathcal{V}^{(r, s)} \quad 8$

$\begin{array}{ll}4.2 \text { Solving } \mathcal{V}^{(r, s)} & 15\end{array}$

$\begin{array}{rlr}4.2 .1 & r=1 & 16\end{array}$

$\begin{array}{lll}4.2 .2 r>1 & 16\end{array}$

5 Conclusions 23

\section{Introduction}

Color-kinematic duality (or BCJ duality) [2] was discovered by Bern, Carrasco and Johansson in 2008. This duality states that Yang-Mills amplitudes can be written in a socalled BCJ formula where kinematic factors share the same algebraic properties (including antisymmetry and Jacobi identity) with color factors. BCJ duality implies relations between color-ordered amplitudes at tree-level. Specifically, the antisymmetry implies KleissKuijf relation (KK relation) [3], while the Jacobi-identity implies Bern-Carrasco-Johansson (BCJ) relation [2]. With these relations, one can reduce the number of independent treelevel color-ordered amplitudes to $(n-3)$ !. Both KK and BCJ relations have been proven in string theory $[4,5]$ and field theory $[6-10]$.

To understand the duality, further efforts including the loop-level BCJ duality [1117, 17-25], the construction of BCJ numerators (by pure spinor string method [26], by kinematic algebra [27-30], with relabeling symmetry [31-33] and from scattering equations [33-37]) as well as the dual trace-factors [33, 38-41] have been made. In another direction, one may wonder whether the BCJ duality and the amplitude relations implied by the duality exist in other theories. An interesting example is the duality and relations in three dimensional supersymmetric theories with 3 -algebra [42]. Another interesting extension is the amplitude relations in nonlinear sigma model with traditional $\mathrm{U}(N)$ Lie algebra [1].

In [1], the authors proved the $\mathrm{U}(1)$ identity and the fundamental BCJ relation for three-level currents with one off-shell leg. The on-shell versions of these two relations 
were obtained by taking on-shell limit of the off-shell leg. Using the method for generating general on-shell KK and BCJ relations by the fundamental ones [43], one can obtain all the general on-shell KK and BCJ relations which have the same formulae with the corresponding relations in Yang-Mills theory.

Although all the on-shell versions of KK and BCJ relations for tree-level amplitudes in nonlinear sigma model have been proven in [1], only two special off-shell relations, namely U(1) identity and fundamental BCJ relation, have been studied. These off-shell relations do not share the same formulae with those in Yang-Mills theory [1]. Actually, in Yang-Mills theory, there have been suggested (all leg) off-shell KK relations [44] which have the same formulae with the corresponding on-shell relations. No BCJ relation for off-shell currents in Yang-Mills theory was found. ${ }^{1}$

A question is whether we can find the full off-shell extensions of the general on-shell KK and BCJ relations in nonlinear sigma model. There are several possible ways to think about this question. One way is to construct the BCJ formula in nonlinear sigma model and apply the algebraic properties to the kinematic factors. The main obstacles for this approach are the infinite number of vertices and the existence of off-shell leg. Another attempt is to generate all off-shell relations from the known off-shell U(1) identity and off-shell fundamental BCJ relation. However, the existence of the off-shell leg again becomes the main trouble.

In this note, we propose a generalized U(1) identity for even-point off-shell currents $J(\sigma)$ in nonlinear sigma model. As already shown in the papers [45, 46], under Cayley parametrization, the odd-point currents (with even numbers of on-shell legs and one offshell leg) have to vanish $[45,46]$. The generalized U(1) identity for even-point currents (with odd numbers of on-shell legs and one off-shell leg) is given by

$$
\begin{gathered}
\sum_{\sigma \in O P\left(\left\{\alpha_{1}, \ldots, \alpha_{r}\right\} \cup\left\{\beta_{1}, \ldots, \beta_{s}\right\}\right)} J(\sigma) \\
=\sum_{\substack{D \in \text { Divisions of }\{\alpha\},\{\beta\} \\
\text { s.t., }\left\{R_{D}-S_{D} \mid=1\right.}}\left(\frac{1}{2 F^{2}}\right)^{\frac{R_{D}+S_{D}-1}{2}} J\left(A_{1}\right) \ldots J\left(A_{R_{D}}\right) J\left(B_{1}\right) \ldots J\left(B_{S_{D}}\right) .
\end{gathered}
$$

On the left hand side of (1.1), we summed over all the ordered permutations $O P(\{\alpha\} \bigcup\{\beta\})$ with keeping the relative orders in each set. For example, in $O P\left(\left\{\alpha_{1}, \alpha_{2}\right\} \bigcup\left\{\beta_{1}, \beta_{2}\right\}\right)$, we have permutations $\left(\alpha_{1}, \alpha_{2}, \beta_{1}, \beta_{2}\right), \quad\left(\alpha_{1}, \beta_{1}, \alpha_{2}, \beta_{2}\right), \quad\left(\alpha_{1}, \beta_{1}, \beta_{2}, \alpha_{2}\right), \quad\left(\beta_{1}, \alpha_{1}, \alpha_{2}, \beta_{2}\right)$, $\left(\beta_{1}, \alpha_{1}, \beta_{2}, \alpha_{2}\right),\left(\beta_{1}, \beta_{2}, \alpha_{1}, \alpha_{2}\right)$. On the right hand side, we have summed over all the possible divisions $D$ of $\{\alpha\}$ and $\{\beta\}$ into ordered subsets $\left\{A_{1}\right\}, \ldots,\left\{A_{R_{D}}\right\}$ and $\left\{B_{1}\right\}, \ldots,\left\{B_{S_{D}}\right\}$ with odd numbers of elements in each subset. The numbers of subsets $R_{D}$ and $S_{D}$ for given division $D$ should satisfy $\left|R_{D}-S_{D}\right|=1$. For example, if we have three elements in the $\{\alpha\}$ set and four elements in the $\{\beta\}$ set, we have

- two $(1,2)$ divisions with $\{\alpha\} \rightarrow\left\{\alpha_{1}, \alpha_{2}, \alpha_{3}\right\}$ and $\{\beta\} \rightarrow\left\{\beta_{1}\right\},\left\{\beta_{2}, \beta_{3}, \beta_{4}\right\}$ or $\{\beta\} \rightarrow$ $\left\{\beta_{1}, \beta_{2}, \beta_{3}\right\},\left\{\beta_{4}\right\}$

- two $(3,2)$ divisions with $\{\alpha\} \rightarrow\left\{\alpha_{1}\right\},\left\{\alpha_{2}\right\},\left\{\alpha_{3}\right\}$ and $\{\beta\} \rightarrow\left\{\beta_{1}\right\},\left\{\beta_{2}, \beta_{3}, \beta_{4}\right\}$ or $\{\beta\} \rightarrow\left\{\beta_{1}, \beta_{2}, \beta_{3}\right\},\left\{\beta_{4}\right\}$

\footnotetext{
${ }^{1}$ Only the off-shell BCJ relation for $\phi^{3}$ colored scalar theory was proposed [44].
} 
- one $(3,4)$ division with $\{\alpha\} \rightarrow\left\{\alpha_{1}\right\},\left\{\alpha_{2}\right\},\left\{\alpha_{3}\right\}$ and $\{\beta\} \rightarrow\left\{\beta_{1}\right\},\left\{\beta_{2}\right\},\left\{\beta_{3}\right\},\left\{\beta_{4}\right\}$.

A special case of the off-shell generalized U(1) identity (1.1) is $r=1$ (or $s=1$ ). In this case, $R, S$ (or $S, R$ ) have to be 1,2 respectively and we arrive at the $\mathrm{U}(1)$ identity proven in [1] (see (2.8)). When multiplying a $p_{1}^{2} \rightarrow 0$ to the right hand side of the relation (1.1), we just arrive at the corresponding on-shell relation for color-ordered amplitudes $A^{2}$

$$
\sum_{\sigma \in O P\left(\left\{\alpha_{1}, \ldots, \alpha_{r}\right\} \bigcup\left\{\beta_{1}, \ldots, \beta_{s}\right\}\right)} A(1,\{\sigma\})=0,
$$

which has been shown to be equivalent with the on-shell KK relation [44].

To prove the off-shell identity (1.1), we first study the eight-point identity with $r=$ $3, s=4$ by explicit calculations with Berends-Giele recursion. Because of the complexity, it seems impossible to extend the calculation directly to a general proof. Instead, we redefine the coefficients for products of subcurrents level by level. After this redefinition, all the divisions $D$ with $R_{D}+S_{D}<r+s$ have the right coefficients in (1.1). Then we only need to prove that the coefficient for $(r, s)$ division has the right form. By combining the $\mathrm{U}(1)$ identity with a generalized U(1) identity with fewer $\alpha$ 's, we obtain a set of equations which are finally used to determine the $(r, s)$ coefficient.

The structure of this note is following. In section 2, we review the Feynman rules, Berends-Giele recursion and the $\mathrm{U}(1)$ identity proved in the paper [1]. In section 3, we study the generalized $\mathrm{U}(1)$ identity with three elements in $\{\alpha\}$ and four elements in $\{\beta\}$ by Berends-Giele recursion directly. It will be quite hard to extend this calculation to a general proof. In section 4 , we provide another approach by redefining the coefficients of divisions with $R_{D}+S_{D}<r+s$ step by step. After these redefinitions, all divisions with $R_{D}+S_{D}<r+s$ already have the right coefficients. We then prove that the coefficient for $(r, s)$ division also has the right form. At last, we conclude this work in section 5 .

\section{Preparation: Feynman rules, Berends-Giele recursion and U(1) iden- tity}

In this section, we review Feynman rules, the Berends-Giele recursion and the U(1) identity in nonlinear sigma model. ${ }^{3}$ Most of the notations follow the recent papers [45, 46].

\subsection{Feynman rules}

Lagrangian. The Lagrangian of $\mathrm{U}(N)$ non-linear sigma model is

$$
\mathcal{L}=\frac{F^{2}}{4} \operatorname{Tr}\left(\partial_{\mu} U \partial^{\mu} U^{\dagger}\right)
$$

where $F$ is a constant. Using Caylay parametrization as in $[45,46]$, the $U$ is defined by

$$
U=1+2 \sum_{n=1}^{\infty}\left(\frac{1}{2 F} \phi\right)^{n},
$$

where $\phi=\sqrt{2} \phi^{a} t^{a}$ and $t^{a}$ are generators of $\mathrm{U}(N)$ Lie algebra.

\footnotetext{
${ }^{2}$ The on-shell generalized U(1) identity in Yang-Mills theory was firstly proposed in [47].

${ }^{3}$ Parts of this section overlap with the section 2 of [1].
} 
Trace form of color decomposition. The full tree amplitudes can be given by trace form decomposition

$$
M\left(1^{a_{1}}, \ldots, n^{a_{n}}\right)=\sum_{\sigma \in S_{n-1}} \operatorname{Tr}\left(T^{a_{1}} T^{a_{\sigma_{2}}} \ldots T^{a_{\sigma_{n}}}\right) A(1, \sigma) .
$$

Since traces have cyclic symmetry, the color-ordered amplitudes $A$ also satisfy cyclic symmetry

$$
A(1,2, \ldots, n)=A(n, 1, \ldots, n-1) .
$$

Feynman rules for color-ordered amplitudes. Vertices in color-ordered Feynman rules under Cayley parametrization (2.2) are

$$
V_{2 n+1}=0, V_{2 n+2}=\left(-\frac{1}{2 F^{2}}\right)^{n}\left(\sum_{i=0}^{n} p_{2 i+1}\right)^{2}=\left(-\frac{1}{2 F^{2}}\right)^{n}\left(\sum_{i=0}^{n} p_{2 i+2}\right)^{2} .
$$

Here, $p_{j}$ denotes the momentum of the leg $j$; momentum conservation has been considered.

\subsection{Berends-Giele recursion}

In the Feynman rules given above, one can construct tree-level currents ${ }^{4}$ through BerendsGiele recursion

$$
\begin{aligned}
J(2, \ldots, n)=\frac{i}{P_{2, n}^{2}} & \sum_{m=4}^{n} \sum_{1=j_{0}<j_{1}<\cdots<j_{m-1}=n} i V_{m}\left(p_{1}=-P_{2, n}, P_{j_{0}+1, j_{1}}, \cdots, P_{j_{m-2}+1, n}\right) \\
& \times \prod_{k=0}^{m-2} J\left(j_{k}+1, \cdots, j_{k+1}\right)
\end{aligned}
$$

where $p_{1}=-P_{2, n}=-\left(p_{2}+p_{3}+\cdots+p_{n}\right)$ is the momentum of the off-shell leg 1 . The starting point of this recursion is $J(2)=J(3)=\cdots=J(n)=1$.

Since there is at least one odd-point vertex for current with odd-point lines (including the off-shell line) and the odd-point vertices are zero, we always have

$$
J(2, \ldots, 2 m+1)=0,
$$

for $(2 m+1)$-point amplitudes. The currents with even points in general are nonzero and are built up by only odd numbers of even-point sub-currents. Since odd-point currents have to vanish, in all following sections of this paper, we just need to discuss on the relations among even-point currents.

\subsection{The off-shell versions of $U(1)$ identity}

In [1], the authors have proven the U(1) identity for off-shell currents in nonlinear sigma model. The identity is

$$
\sum_{\sigma \in O P\left(\left\{\alpha_{1}\right\} \bigcup\left\{\beta_{1}, \ldots, \beta_{2 m}\right\}\right)} J(\sigma)=\frac{1}{2 F^{2}} \sum_{\text {divisions }\{\beta\} \rightarrow\left\{B_{1}\right\},\left\{B_{2}\right\}} J\left(B_{1}\right) J\left(B_{2}\right),
$$

\footnotetext{
${ }^{4}$ In this paper, an $n$-point current is mentioned as the current with $n-1$ on-shell legs and one off-shell leg.
} 


\begin{tabular}{|c|c|c|c|}
\hline Divisions & Type-1 & Type-2 & Type-3 \\
\hline$\left\{\alpha_{1}\right\}\left\{\alpha_{2}\right\}\left\{\alpha_{3}\right\}\left\{\beta_{1}\right\}\left\{\beta_{2}\right\}\left\{\beta_{3}\right\}\left\{\beta_{4}\right\}$ & $s_{\alpha_{1} \alpha_{3}}+s_{\beta_{1} \beta_{3}}+s_{\beta_{2} \beta_{4}}$ & 0 & $p_{1}^{2}$ \\
\hline$\left\{\alpha_{1}\right\}\left\{\alpha_{2}\right\}\left\{\alpha_{3}\right\}\left\{\beta_{1}, \beta_{2}, \beta_{3}\right\}\left\{\beta_{4}\right\}$ & $-s_{\alpha_{1} \alpha_{3}}$ & $-\left(p_{\beta_{1}}+p_{\beta_{2}}+p_{\beta_{3}}\right)^{2}$ & $p_{1}^{2}$ \\
\hline$\left\{\alpha_{1}\right\}\left\{\alpha_{2}\right\}\left\{\alpha_{3}\right\}\left\{\beta_{1}\right\}\left\{\beta_{2}, \beta_{3}, \beta_{4}\right\}$ & $-s_{\alpha_{1} \alpha_{3}}$ & $-\left(p_{\beta_{2}}+p_{\beta_{3}}+p_{\beta_{4}}\right)^{2}$ & $p_{1}^{2}$ \\
\hline$\left\{\alpha_{1}, \alpha_{2}, \alpha_{3}\right\}\left\{\beta_{1}\right\}\left\{\beta_{2}\right\}\left\{\beta_{3}\right\}\left\{\beta_{4}\right\}$ & $-s_{\beta_{1} \beta_{3}}-s_{\beta_{2} \beta_{4}}$ & $-\left(p_{\alpha_{1}}+p_{\alpha_{2}}+p_{\alpha_{3}}\right)^{2}$ & 0 \\
\hline$\left\{\alpha_{1}, \alpha_{2}, \alpha_{3}\right\}\left\{\beta_{1}, \beta_{2}, \beta_{3}\right\}\left\{\beta_{4}\right\}$ & 0 & $\left(p_{\alpha_{1}}+p_{\alpha_{2}}+p_{\alpha_{3}}\right)^{2}+\left(p_{\beta_{1}}+p_{\beta_{2}}+p_{\beta_{3}}\right)^{2}$ & $p_{1}^{2}$ \\
\hline$\left\{\alpha_{1}, \alpha_{2}, \alpha_{3}\right\}\left\{\beta_{1}\right\}\left\{\beta_{2}, \beta_{3}, \beta_{4}\right\}$ & 0 & $\left(p_{\alpha_{1}}+p_{\alpha_{2}}+p_{\alpha_{3}}\right)^{2}+\left(p_{\beta_{2}}+p_{\beta_{3}}+p_{\beta_{4}}\right)^{2}$ & $p_{1}^{2}$ \\
\hline
\end{tabular}

Table 1. The coefficients of eight-point case in general can be classified into three types. Here we omit the coupling constants for convenience.

where on the left hand side, we summed over the permutations in $\left\{\alpha_{1}\right\} \bigcup\left\{\beta_{1}, \ldots, \beta_{2 m}\right\}$ with keeping the relative order in the $\beta$ set. On the right hand side, we summed over the divisions of $\{\beta\}$ into two ordered subsets.

\section{Direct calculation of an eight-point example}

We have checked the generalized U(1) identity (1.1) for four- and six-point currents. In the four-point case, we only have $r=1, s=2$ and $r=2, s=1$, which are $\mathrm{U}(1)$ identities (2.8). In the six-point case, $r=1, s=4$ and $r=4, s=1$ are also U(1) identities (2.8). The new relations for six-point currents are the cases with $r=2, s=3$ and $r=3, s=2$, where the later case can be obtained from the former one by exchanging the roles of $\alpha$ and $\beta$. We just skip all the calculations of four- and six-point identities and show a more complicated eight-point example.

We take the eight-point identity with three elements in $\{\alpha\}$ and four elements in $\{\beta\}$ as an example. The explicit form of the identity (1.1) with $r=3, s=4$ is

$$
\begin{aligned}
& \sum_{\sigma \in O P\left(\left\{\alpha_{1}, \alpha_{2}, \alpha_{3}\right\} \bigcup\left\{\beta_{1}, \beta_{2}, \beta_{3}, \beta_{4}\right\}\right)} J(\sigma) \\
= & \frac{1}{2 F^{2}}\left[J\left(\alpha_{1}, \alpha_{2}, \alpha_{3}\right) J\left(\beta_{1}\right) J\left(\beta_{2}, \beta_{3}, \beta_{4}\right)+J\left(\alpha_{1}, \alpha_{2}, \alpha_{3}\right) J\left(\beta_{1}, \beta_{2}, \beta_{3}\right) J\left(\beta_{4}\right)\right] \\
& +\left(\frac{1}{2 F^{2}}\right)^{2}\left[J\left(\alpha_{1}\right) J\left(\alpha_{2}\right) J\left(\alpha_{3}\right) J\left(\beta_{1}\right) J\left(\beta_{2}, \beta_{3}, \beta_{4}\right)+J\left(\alpha_{1}\right) J\left(\alpha_{2}\right) J\left(\alpha_{3}\right) J\left(\beta_{1}, \beta_{2}, \beta_{3}\right) J\left(\beta_{4}\right)\right] \\
& +\left(\frac{1}{2 F^{2}}\right)^{3} J\left(\alpha_{1}\right) J\left(\alpha_{2}\right) J\left(\alpha_{3}\right) J\left(\beta_{1}\right) J\left(\beta_{2}\right) J\left(\beta_{3}\right) J\left(\beta_{4}\right) .
\end{aligned}
$$

To prove this identity, we use Berends-Giele recursion (2.6) to express all the currents on the left hand side of (3.1) by six- and four-point subcurrents. Then we collect the terms with a same vertex connected to the off-shell leg 1. After summing all the possible diagrams in each collection, the left hand side of (3.1) is expressed by

- diagrams containing six-point and (or) four-point substructures of generalized U(1)identity (see figure 1(A))

- diagrams with neither six-point nor four-point substructure (see figure 1(B)). 


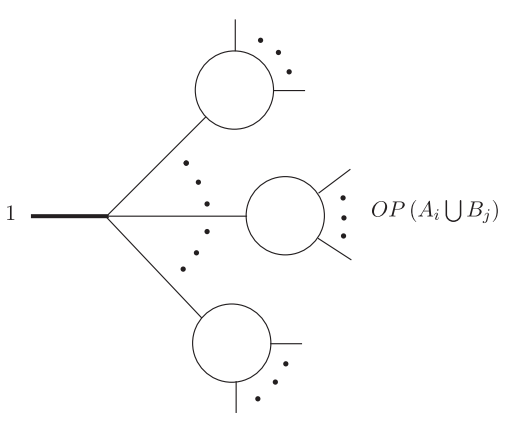

(A)

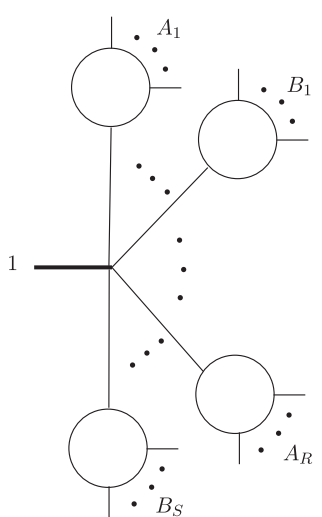

(B)

Figure 1. Two classes of diagrams: (A) diagrams containing substructures of generalized U(1) identity such as $\sigma \in O P\left(A_{i} \cup B_{j}\right)$ where $A_{i}$ and $B_{j}$ denote ordered subsets of $\{\alpha\}$ and $\{\beta\}$. (B) diagrams with each subcurrent containing only $\{\alpha\}$ elements or $\{\beta\}$ elements.

Remembering that the identity (1.1) is satisfied by four- and six-point currents, we apply these lower-point identities to the four- and six-point substructures in the first class of diagram. Then diagrams in the first class are rewritten in terms of products of subcurrents containing only $\alpha$ or $\beta$ elements. Since the second class of diagram does not have any substructure, it is already expressed by products of subcurrents containing only $\alpha$ or $\beta$ elements. After this reduction, for a given product of subcurrents (or in other words, given division of $\{\alpha\}$ set and $\{\beta\}$ set), we collect the coefficients together. Thus the left hand side of (3.1) is written as

$$
\begin{aligned}
& \left(\frac{1}{2 F^{2}}\right)^{3}\left[\left(s_{\alpha_{1} \alpha_{3}}+s_{\beta_{1} \beta_{3}}+s_{\beta_{2} \beta_{4}}\right)+p_{1}^{2}\right] \frac{1}{p_{1}^{2}} J\left(\alpha_{1}\right) J\left(\alpha_{2}\right) J\left(\alpha_{3}\right) J\left(\beta_{1}\right) J\left(\beta_{2}\right) J\left(\beta_{3}\right) J\left(\beta_{4}\right) \\
& +\left(\frac{1}{2 F^{2}}\right)^{2}\left[-s_{\alpha_{1} \alpha_{3}}-\left(p_{\beta_{1}}+p_{\beta_{2}}+p_{\beta_{3}}\right)^{2}+p_{1}^{2}\right] \frac{1}{p_{1}^{2}} J\left(\alpha_{1}\right) J\left(\alpha_{2}\right) J\left(\alpha_{3}\right) J\left(\beta_{1}, \beta_{2}, \beta_{3}\right) J\left(\beta_{4}\right) \\
& +\left(\frac{1}{2 F^{2}}\right)^{2}\left[-s_{\alpha_{1} \alpha_{3}}-\left(p_{\beta_{2}}+p_{\beta_{3}}+p_{\beta_{4}}\right)^{2}+p_{1}^{2}\right] \frac{1}{p_{1}^{2}} J\left(\alpha_{1}\right) J\left(\alpha_{2}\right) J\left(\alpha_{3}\right) J\left(\beta_{1}\right) J\left(\beta_{2}, \beta_{3}, \beta_{4}\right) \\
& +\left(\frac{1}{2 F^{2}}\right)^{2}\left[\left(-s_{\beta_{1} \beta_{3}}-s_{\beta_{2} \beta_{4}}\right)-\left(p_{\alpha_{1}}+p_{\alpha_{2}}+p_{\alpha_{3}}\right)^{2}\right] \frac{1}{p_{1}^{2}} J\left(\alpha_{1}, \alpha_{2}, \alpha_{3}\right) J\left(\beta_{1}\right) J\left(\beta_{2}\right) J\left(\beta_{3}\right) J\left(\beta_{4}\right) \\
& +\left(\frac{1}{2 F^{2}}\right)\left[\left(p_{\alpha_{1}}+p_{\alpha_{2}}+p_{\alpha_{3}}\right)^{2}+\left(p_{\beta_{1}}+p_{\beta_{2}}+p_{\beta_{3}}\right)^{2}+p_{1}^{2}\right] \frac{1}{p_{1}^{2}} J\left(\alpha_{1}, \alpha_{2}, \alpha_{3}\right) J\left(\beta_{1}, \beta_{2}, \beta_{3}\right) J\left(\beta_{4}\right) \\
& +\left(\frac{1}{2 F^{2}}\right)\left[\left(p_{\alpha_{1}}+p_{\alpha_{2}}+p_{\alpha_{3}}\right)^{2}+\left(p_{\beta_{2}}+p_{\beta_{3}}+p_{\beta_{4}}\right)^{2}+p_{1}^{2}\right] \frac{1}{p_{1}^{2}} J\left(\alpha_{1}, \alpha_{2}, \alpha_{3}\right) J\left(\beta_{1}\right) J\left(\beta_{2}, \beta_{3}, \beta_{4}\right),
\end{aligned}
$$

where $s_{i j} \equiv\left(p_{i}+p_{j}\right)^{2}$. Coefficients for each division can be classified into three types (see 1). A type-2 coefficient always cancels with a propagator of a subcurrent and divides the subcurrent into new subcurrents. For example, the coefficient in type- 2 term on the second line is $-\left(p_{\beta_{1}}+p_{\beta_{2}}+p_{\beta_{3}}\right)^{2}$ which reduce the current $J\left(\beta_{1}, \beta_{2}, \beta_{3}\right)$ to 


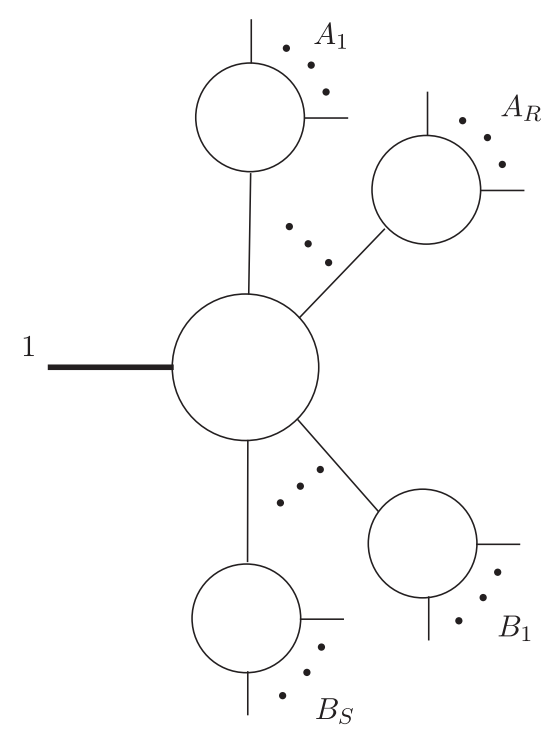

Figure 2. We redefine the coefficients for $R+S<r+s$ divisions such that they are the right ones as in the general form (1.1). Then we solve the $R=r, S=s$ coefficient.

$-\left(\frac{1}{2 F^{2}}\right) s_{\beta_{1} \beta_{3}} J\left(\beta_{1}\right) J\left(\beta_{2}\right) J\left(\beta_{3}\right)$. Thus this part of contribution cancels with the second term of the type- 1 coefficient of $(3,4)$ division. Similarly, other type- 2 terms also cancel with type- 1 terms for divisions with larger $R_{D}+S_{D}$. All the type- 1 and type- 2 terms cancel out in this way. Only the type-3 terms are left and give the right hand side of the eight-point identity (3.1).

\section{Proof of the generalized U(1) identity for off-shell currents}

In the previous section, we have provided a direct approach to an eight-point example by Berends-Giele recursion. Although the coefficients in the example were shown to have a good pattern (see table 1), it will be quite hard to extend the calculation to a general proof. One reason is that we will encounter many different lower-point substructures of the identity (1.1) when the number of $\{\alpha\}$ elements grows. Thus we have to prove the general formula (1.1) in a different way. In this section, we will show a general proof of the identity (1.1). The main idea is following:

- As we have done in the eight-point example, we write the left hand side of the identity (1.1) by Berends-Giele recursion and collect the diagrams with a same vertex attached to the off-shell leg 1 (See figure 1(A) and (B)). Reducing the substructures by lower-point identities and putting the coefficients corresponding to each product of subcurrents together, we express the left hand side of (1.1) as follows

$$
\begin{aligned}
& \sum_{\sigma \in O P\left(\left\{\alpha_{1}, \ldots, \alpha_{r}\right\}\right.} J(\sigma) \\
& =\sum_{D \in \text { Divisions }} \frac{1}{p_{1}^{2}}\left[\sum_{\left.\left.i_{1}, \ldots, \beta_{s}\right\}\right)} V_{4}^{i_{4, D}}-\sum_{i_{6, D}} V_{6}^{i_{6, D}}+\sum_{i_{8, D}} V_{8}^{i_{8, D}}-\cdots+(-1)^{\frac{R_{D}+S_{D}-1}{2}} \sum_{i_{R_{D}+S_{D}+1, D}} V_{R_{D}+S_{D}+1}^{i_{R_{2}}+S_{D}+1, D}\right]
\end{aligned}
$$




$$
\times J\left(A_{1}\right) \ldots J\left(A_{R_{D}}\right) J\left(B_{1}\right) \ldots J\left(B_{S_{D}}\right),
$$

where $V_{l}^{i_{l, D}}$ denote the $l$-point vertices which contribute to the division $D$ and $\sum_{i_{4, D}}$ means that we sum over all such $l$-point vertices. The prefactor $(-1)^{\frac{l-1}{2}}$ of $l$-point vertex comes from the factor $\left(-\frac{1}{2 F^{2}}\right)^{n}$ in the Feynman rules $(2.5)$.

- We show that the expression obtained in the above step can be rearranged (figure 2) into the following formula

$$
\begin{gathered}
\sum_{\sigma \in O P\left(\left\{\alpha_{1}, \ldots, \alpha_{r}\right\} \bigcup\left\{\beta_{1}, \ldots, \beta_{s}\right\}\right)} J(\sigma) \\
=\sum_{\substack{D \in \text { Divisions of }_{R_{D}+S_{D}<r+s}\left\{,\{\beta\} \\
R^{2}\right.}}\left(\frac{1}{2 F^{2}}\right)^{\frac{R_{D}+S_{D}-1}{2}} \delta\left(\left|R_{D}-S_{D}\right|-1\right) J\left(A_{1}\right) \ldots J\left(A_{R_{D}}\right) J\left(B_{1}\right) \ldots J\left(B_{S_{D}}\right) \\
\\
\quad+\left(\frac{1}{2 F^{2}}\right)^{\frac{r+s-1}{2}} \mathcal{V}^{(r, s)} J\left(\alpha_{1}\right) \ldots J\left(\alpha_{r}\right) J\left(\beta_{1}\right) \ldots J\left(\beta_{s}\right),
\end{gathered}
$$

where $\mathcal{V}^{(r, s)}$ is the dimensionless coefficient for the $(r, s)$ division. The first term of (4.2) is given by sum of divisions $D\left(R_{D}+S_{D}<r+s\right)$ which already have the correct coefficients in (1.1). Thus we only need to prove that the coefficient $\mathcal{V}^{(r, s)}$ in the second term of (4.2) also has the right expression in (1.1), i.e., $\mathcal{V}^{(r, s)}=\delta(|r-s|-1)$.

- The undetermined coefficient $\mathcal{V}^{(r, s)}$ has the general form $\frac{1}{p_{1}^{2}}\left(\sum_{i, j} c_{i j} s_{i j}\right)$ with appropriate $c_{i j}$. By combining an U(1) identity and a generalized U(1) identity with fewer $\alpha$ 's, we can prove that $\mathcal{V}^{(r, s)}=\delta(|r-s|-1)$. Therefore, the generalized U(1) identity (1.1) for off-shell currents is proved.

In the remainder of this section, we will show the left hand side of (1.1) can be rearranged into (4.2) and then solve $\mathcal{V}^{(r, s)}$.

\subsection{Proof of the validity of (4.2) with an undetermined coefficient $\mathcal{V}^{(r, s)}$}

Now we show that the left hand side of the off-shell generalized U(1) identity (1.1) can be rearranged into the form (4.2). We start from several examples.

Four-point example. The four-point example is the four-point U(1) identity (see [1]). By explicit calculation, this is given by the sum of three diagrams in figure 3, i.e.,

$$
\sum_{\sigma \in O P\left(\left\{\alpha_{1}\right\} \bigcup\left\{\beta_{1}, \beta_{2}\right\}\right)} J(\sigma)=\left(\frac{1}{2 F^{2}}\right) J\left(\alpha_{1}\right) J\left(\beta_{1}\right) J\left(\beta_{2}\right) .
$$

The identity with two $\alpha$ 's and one $\beta$ can be obtained by exchanging the roles of $\alpha$ and $\beta$.

Before giving the next example, let us have a look at an off-shell extension of the right hand side of (4.3), i.e., we replace the three on-shell legs $\alpha_{1}, \beta_{1}$ and $\beta_{2}$ in figure 3 by three off-shell currents $J\left(A_{1}\right), J\left(B_{1}\right)$ and $J\left(B_{2}\right)$ correspondingly. From Feynman rules (2.5), 


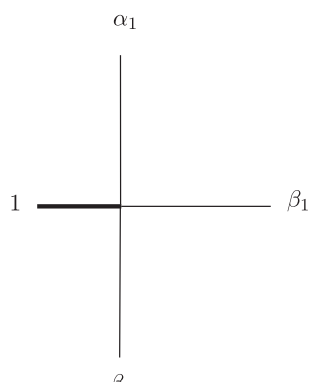

$\beta_{2}$

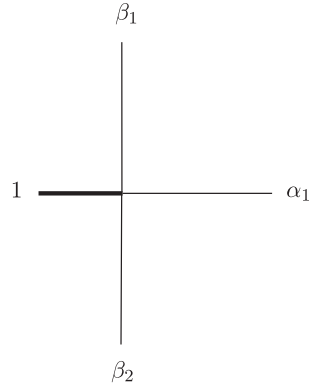

$\beta_{2}$

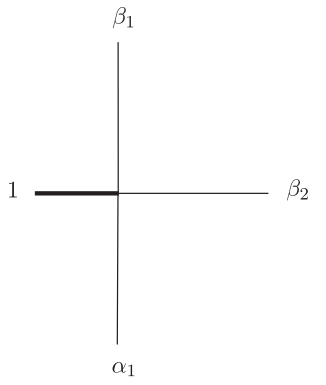

$\alpha_{1}$

Figure 3. Diamgrams contributing to four-point identity.

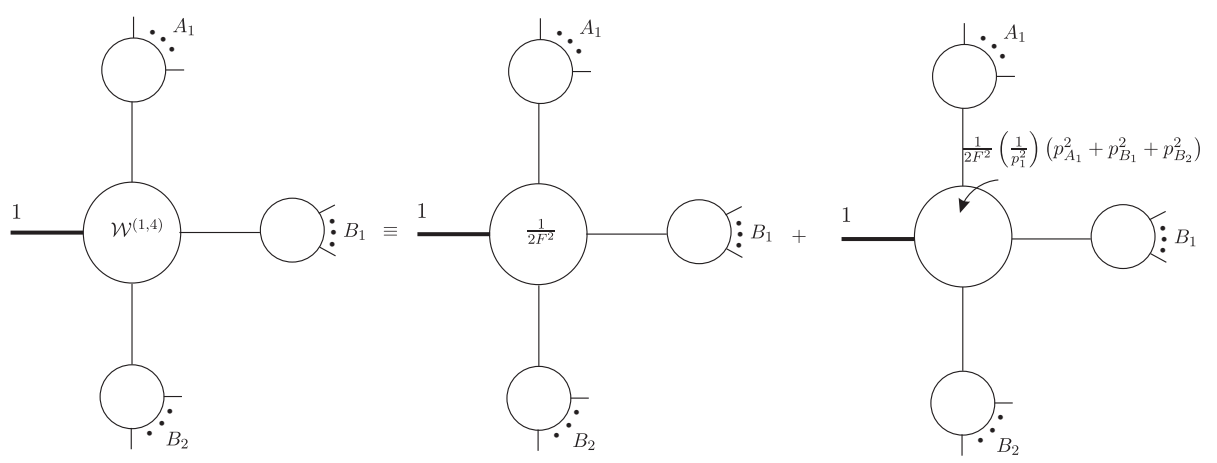

Figure 4. The off-shell extension of four-point identity. Here we absorb the $\frac{1}{p_{1}^{2}}$ corresponding to the off-shell leg 1 into the coefficients for convenience.

the coefficient of $J\left(A_{1}\right) J\left(B_{1}\right) J\left(B_{2}\right)$ is written as a linear combination of $\frac{1}{p_{1}^{2}}\left(p_{i} \cdot p_{j}\right)$, where $i, j$ can be either one of $A_{1}, B_{1}, B_{2}$; we use $p_{A_{i}}, p_{B_{i}}$ to denote the sum of momenta of elements in $A_{i}, B_{i}$ respectively. Then we consider the sum of diagrams with the off-shell leg 1 connected to a four-point vertex whose other three legs are attached to the currents $J\left(A_{1}\right), J\left(B_{1}\right)$ and $J\left(B_{2}\right)$. In general, we should have

$$
\sum_{\sigma \in O P\left(\left\{A_{1}\right\} \bigcup\left\{B_{1}, B_{2}\right\}\right)} J^{(4)}(\sigma)=\mathcal{W}^{(1,2)} J\left(A_{1}\right) J\left(B_{1}\right) J\left(B_{2}\right),
$$

with

$$
\mathcal{W}^{(1,2)} \equiv\left(\frac{1}{2 F^{2}}\right)\left[1+\frac{1}{p_{1}^{2}}\left(a_{1}^{(1,2)} p_{A_{1}}^{2}+b_{1}^{(1,2)} p_{B_{1}}^{2}+b_{2}^{(1,2)} p_{B_{2}}^{2}\right)\right]
$$

Here, $J^{(4)}(\sigma)$ denote the diagrams with the four-point vertices connected to $1, J\left(A_{1}\right), J\left(B_{1}\right)$ and $J\left(B_{2}\right)$ and $a_{1}^{(1,2)}, b_{1}^{(1,2)}$ and $b_{2}^{(1,2)}$ are some constant coefficients. The equation (4.4) is the only possible formula of all-leg-off-shell extension of the four-point identity (4.3) for oneleg-off-shell currents. This is because when replacing the currents $J\left(A_{1}\right), J\left(B_{1}\right)$ and $J\left(B_{2}\right)$ by on shell legs $\alpha_{1}, \beta_{1}$ and $\beta_{2}$, we have to return to (4.3). The coefficient thus can only be the sum of $\left(\frac{1}{2 F^{2}}\right)$ and combinations of $\frac{1}{p_{1}^{2}} p_{A_{i}}^{2}, \frac{1}{p_{1}^{2}} p_{B_{i}}^{2}$, which vanish under on-shell limit. From the explicit calculation in [1], we can see $a_{1}^{(1,2)}=b_{1}^{(1,2)}=b_{2}^{(1,2)}=1$. Thus the off-shell extension (4.5) can be expressed by figure 4. We will encounter (4.4), (4.5) in higher-point cases. 


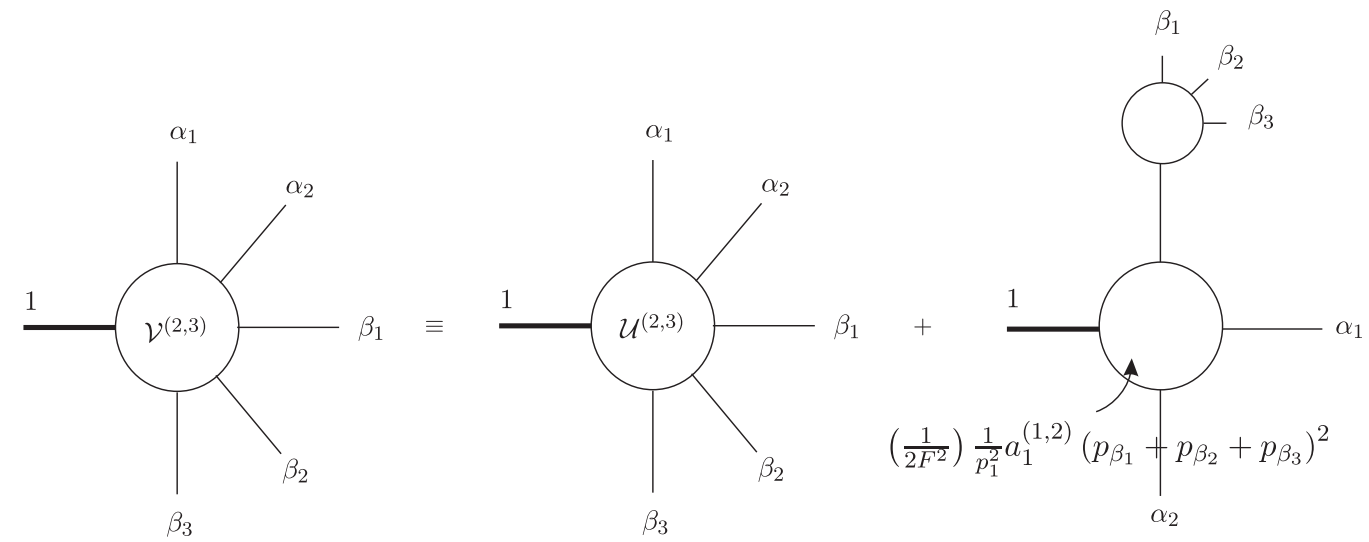

Figure 5. Redefinition of the coefficient of $(2,3)$ division for the identity with two $\alpha$ 's and three $\beta$ 's.

Six-point example. With the four-point identity in hand, let us consider the six-point example. The first six-point example is the $\mathrm{U}(1)$-identity with only one $\alpha$, which has been understood. Now we consider the generalized identity with two $\alpha$ 's

$$
\begin{aligned}
\sum_{\sigma \in O P\left(\left\{\alpha_{1}, \alpha_{2}\right\} \bigcup\left\{\beta_{1}, \beta_{2}, \beta_{3}\right\}\right)} J(\sigma)= & \frac{1}{2 F^{2}} J\left(\alpha_{1}\right) J\left(\alpha_{2}\right) J\left(\beta_{1}, \beta_{2}, \beta_{3}\right) \\
& +\left(\frac{1}{2 F^{2}}\right)^{2} J\left(\alpha_{1}\right) J\left(\alpha_{2}\right) J\left(\beta_{1}\right) J\left(\beta_{2}\right) J\left(\beta_{3}\right) .
\end{aligned}
$$

Step-1. To prove this identity, we start from the left hand side. We use Berends-Giele recursion to express the currents on the left hand side. Then collect the diagrams together with same substructures of generalized U(1)-identity. After reducing diagrams containing four-point substructures of U(1)-identity by (4.3), we collect the coefficients for given division of $\{\alpha\}$ and $\{\beta\}$. Then the left hand side of the six-point identity has the form

$$
\begin{aligned}
\sum_{\sigma \in O P\left(\left\{\alpha_{1}, \alpha_{2}\right\} \bigcup\left\{\beta_{1}, \beta_{2}, \beta_{3}\right\}\right)} J(\sigma)= & \mathcal{U}_{1}^{(2,3)} J\left(\alpha_{1}\right) J\left(\alpha_{2}\right) J\left(\beta_{1}\right) J\left(\beta_{2}\right) J\left(\beta_{3}\right) \\
& +\mathcal{U}_{2}^{(2,3)} J\left(\alpha_{1}\right) J\left(\alpha_{2}\right) J\left(\beta_{1}, \beta_{2}, \beta_{3}\right),
\end{aligned}
$$

with $\mathcal{U}_{1}^{(2,3)}$ and $\mathcal{U}_{2}^{(2,3)}$ as coefficients. In general, $\mathcal{U}_{1}^{(2,3)}$ and $\mathcal{U}_{2}^{(2,3)}$ are written as sum of terms of the form $\frac{1}{p_{1}^{2}}\left(p_{i} \cdot p_{j}\right)$, where $\frac{1}{p_{1}^{2}}$ and $\left(p_{i} \cdot p_{j}\right)$ respectively come from the off-shell propagator and vertices (as shown in (4.1)). The second term in $(4.7)$ is the $(2,1)$ division which can only get contribution from diagrams with the off-shell leg 1 directly connected to four-point vertices whose other three lines are connected to $J\left(\alpha_{1}\right), J\left(\alpha_{2}\right)$ and $J\left(\beta_{1}, \beta_{2}, \beta_{3}\right)$. The sum of such contributions is noting but the off-shell extension (4.4) in the four-point example with $A_{1} \rightarrow\left\{\beta_{1}, \beta_{2}, \beta_{3}\right\}, B_{1} \rightarrow\left\{\alpha_{1}\right\}, B_{2} \rightarrow\left\{\alpha_{2}\right\}$. Thus we have

$$
\mathcal{U}_{2}^{(2,3)}=\left(\frac{1}{2 F^{2}}\right)\left\{1+\frac{1}{p_{1}^{2}}\left[a_{1}^{(1,2)}\left(p_{\beta_{1}}+p_{\beta_{2}}+p_{\beta_{3}}\right)^{2}\right]\right\}
$$

where the on-shell conditions of $\alpha_{1}$ and $\alpha_{2}$ have been used. 


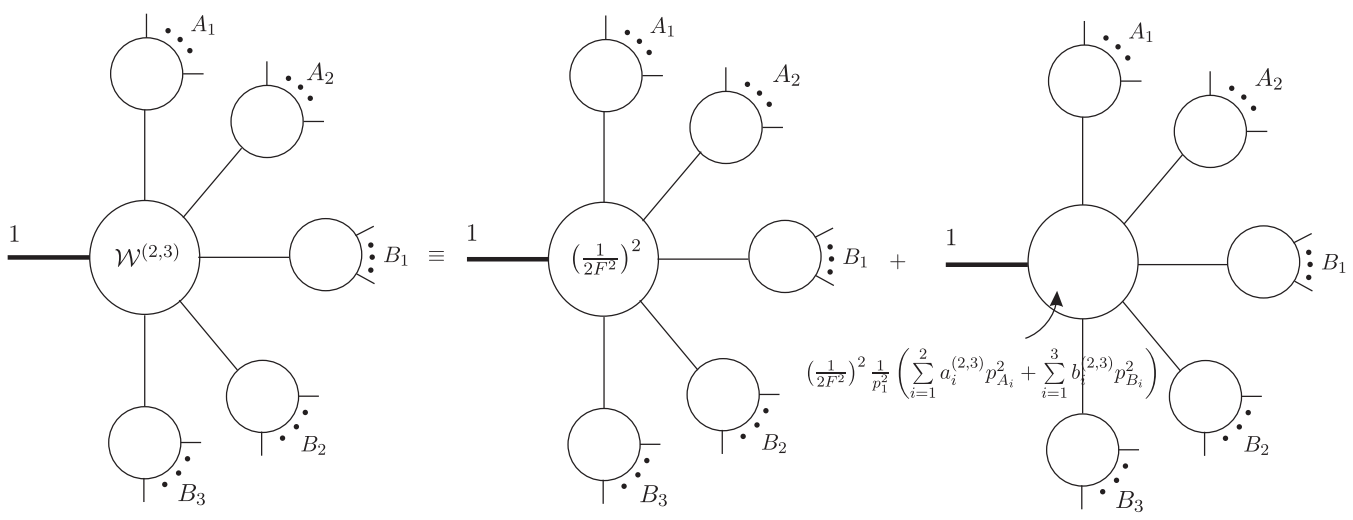

Figure 6. The off-shell extension of the $(2,3)$ division in the six-point identity with two $\alpha$ 's and three $\beta$ 's.

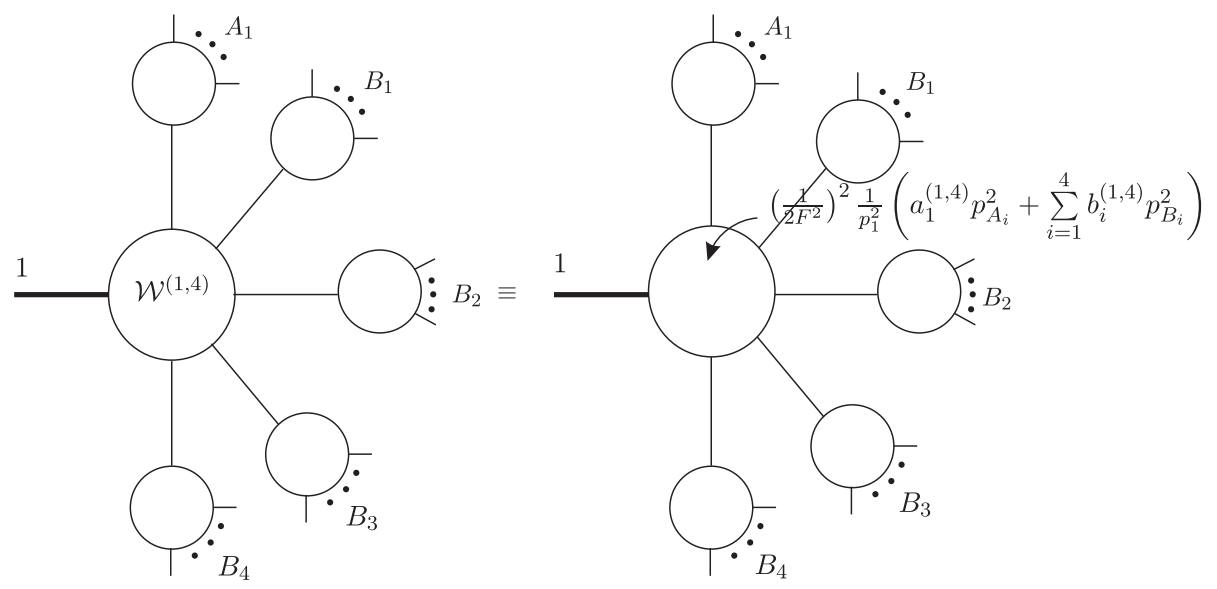

Figure 7. The off-shell extension of the $(1,4)$ division in the six-point identity with one $\alpha$ and four $\beta$ 's.

Step-2. Since $\left(p_{\beta_{1}}+p_{\beta_{2}}+p_{\beta_{3}}\right)^{2}$ further reduces $J\left(\beta_{1}, \beta_{2}, \beta_{3}\right)$ to $J\left(\beta_{1}\right) J\left(\beta_{2}\right) J\left(\beta_{3}\right)$ with a coefficient $\left(\frac{1}{2 F^{2}}\right) s_{\beta_{1} \beta_{3}}$, we rearrange $(4.7)$ by absorbing the term proportional to $\left(p_{\beta_{1}}+p_{\beta_{2}}+p_{\beta_{3}}\right)^{2}$ into $\mathcal{U}_{1}^{(2,3)}$, the left hand side of (4.7) becomes

$$
\begin{aligned}
\sum_{\sigma \in O P\left(\left\{\alpha_{1}, \alpha_{2}\right\} \bigcup\left\{\beta_{1}, \beta_{2}, \beta_{3}\right\}\right)} J(\sigma)= & \mathcal{V}^{(2,3)} J\left(\alpha_{1}\right) J\left(\alpha_{2}\right) J\left(\beta_{1}\right) J\left(\beta_{2}\right) J\left(\beta_{3}\right) \\
& +\left(\frac{1}{2 F^{2}}\right) J\left(\alpha_{1}\right) J\left(\alpha_{2}\right) J\left(\beta_{1}, \beta_{2}, \beta_{3}\right),
\end{aligned}
$$

where

$$
\mathcal{V}^{(2,3)} \equiv \mathcal{U}_{1}^{(2,3)}+a_{1}^{(1,2)}\left(\frac{1}{2 F^{2}}\right)^{2} \frac{1}{p_{1}^{2}} s_{\beta_{1} \beta_{3}}
$$

which is shown by figure (5). The new defined coefficient of $(2,1)$ division is what we want. We need to prove $\mathcal{V}^{(2,3)}=\left(\frac{1}{2 F^{2}}\right)^{2}$ for the $(2,3)$ division. In the next subsection, we have a general proof of this. 
Let us consider the off-shell extension of the first term on the right hand side of (4.9), assuming that we have already proved $\mathcal{V}^{(2,3)}=\left(\frac{1}{2 F^{2}}\right)^{2}$. If all the $\alpha$ 's and $\beta$ 's are allowed to be off-shell, we replace $J\left(\alpha_{i}\right)$ by $J\left(A_{i}\right)$ and $J\left(\beta_{i}\right)$ by $J\left(B_{i}\right)$. Recalling that the coefficient of the off-shell extension should return to $\mathcal{V}^{(2,3)}=\left(\frac{1}{2 F^{2}}\right)^{2}$ under the replacement $J\left(A_{i}\right) \rightarrow$ $\alpha_{i}, J\left(B_{i}\right) \rightarrow \beta_{i}$ and $\mathcal{V}^{(2,3)}$ can only be of the form $\frac{1}{p_{1}^{2}} \sum_{i j} c_{i j} p_{i} \cdot p_{j}$, the off-shell extension of $\mathcal{V}^{(2,3)}$ must have the form (see figure 6)

$$
\mathcal{W}^{(2,3)} \equiv\left(\frac{1}{2 F^{2}}\right)^{2}\left[1+\frac{1}{p_{1}^{2}}\left(\sum_{i=1}^{2} a_{i}^{(2,3)} p_{A}^{2}+\sum_{i=1}^{3} b_{i}^{(2,3)} p_{B}^{2}\right)\right] .
$$

Following a parallel discussion, we can do the same on the six-point relation with only one $\alpha$ and extend the coefficient of $(1,4)$ division to off-shell case (see figure 7 )

$$
\mathcal{W}^{(1,4)} \equiv\left(\frac{1}{2 F^{2}}\right)^{2} \frac{1}{p_{1}^{2}}\left(a_{1}^{(1,4)} p_{A}^{2}+\sum_{i=1}^{4} b_{i}^{(1,4)} p_{B}^{2}\right) .
$$

Eight-point example. We now consider an eight-point example with three $\alpha$ 's and five $\beta$ 's. The formula of this example is given by (3.1) in section 3 .

Step-1. To prove the eight-point example, we first express the left hand side of (3.1) by Berends-Giele recursion and then collect the contributions to a substructure of generalized U(1)-identity together. After applying generalized U(1)-identity, we get

$$
\begin{aligned}
& \sum_{\sigma \in O P\left(\left\{\alpha_{1}, \alpha_{2}, \alpha_{3}\right\} \bigcup\left\{\beta_{1}, \beta_{2}, \beta_{3}, \beta_{4}\right\}\right)} J(\sigma) \\
= & \mathcal{U}_{1}^{(3,4)} J\left(\alpha_{1}\right) J\left(\alpha_{2}\right) J\left(\alpha_{3}\right) J\left(\beta_{1}\right) J\left(\beta_{2}\right) J\left(\beta_{3}\right) J\left(\beta_{4}\right)+\mathcal{U}_{2}^{(3,4)} J\left(\alpha_{1}\right) J\left(\alpha_{2}\right) J\left(\alpha_{3}\right) J\left(\beta_{1}, \beta_{2}, \beta_{3}\right) J\left(\beta_{4}\right) \\
& +\mathcal{U}_{3}^{(3,4)} J\left(\alpha_{1}\right) J\left(\alpha_{2}\right) J\left(\alpha_{3}\right) J\left(\beta_{1}\right) J\left(\beta_{2}, \beta_{3}, \beta_{4}\right)+\mathcal{U}_{4}^{(3,4)} J\left(\alpha_{1}, \alpha_{2}, \alpha_{3}\right) J\left(\beta_{1}\right) J\left(\beta_{2}\right) J\left(\beta_{3}\right) J\left(\beta_{4}\right) \\
& +\mathcal{U}_{5}^{(3,4)} J\left(\alpha_{1}, \alpha_{2}, \alpha_{3}\right) J\left(\beta_{1}, \beta_{2}, \beta_{3}\right) J\left(\beta_{4}\right)+\mathcal{U}_{6}^{(3,4)} J\left(\alpha_{1}, \alpha_{2}, \alpha_{3}\right) J\left(\beta_{1}\right) J\left(\beta_{2}, \beta_{3}, \beta_{4}\right) .
\end{aligned}
$$

Again, we start from the $R+S=3$ divisions, there are two cases corresponding to the last two terms of the above equation. These cases only get contributions from diagrams with the off-shell leg 1 connected to a four-point vertex. As shown in the six-point example, the coefficients $\mathcal{U}_{5}$ and $\mathcal{U}_{6}$ can be given by the off-shell extension $\mathcal{W}^{(1,2)}$ (figure 4 ), particularly

$$
\mathcal{U}_{5}^{(3,4)}=\left(\frac{1}{2 F^{2}}\right) \frac{1}{p_{1}^{2}}\left[p_{1}^{2}+a_{1}^{(1,2)}\left(p_{\alpha_{1}}+p_{\alpha_{2}}+p_{\alpha_{3}}\right)^{2}+b_{1}^{(1,2)}\left(p_{\beta_{1}}+p_{\beta_{2}}+p_{\beta_{3}}\right)^{2}\right]
$$

and

$$
\mathcal{U}_{6}^{(3,4)}=\left(\frac{1}{2 F^{2}}\right) \frac{1}{p_{1}^{2}}\left[p_{1}^{2}+a_{1}^{(1,2)}\left(p_{\alpha_{1}}+p_{\alpha_{2}}+p_{\alpha_{3}}\right)^{2}+b_{2}^{(1,2)}\left(p_{\beta_{2}}+p_{\beta_{3}}+p_{\beta_{4}}\right)^{2}\right] .
$$

Step-2. The term $\left(p_{\alpha_{1}}+p_{\alpha_{2}}+p_{\alpha_{3}}\right)^{2}$ in $\mathcal{U}_{5}^{(3,4)}$ and $\mathcal{U}_{6}^{(3,4)}$ reduces $J\left(\alpha_{1}, \alpha_{2}, \alpha_{3}\right)$ to $J\left(\alpha_{1}\right) J\left(\alpha_{2}\right) J\left(\alpha_{3}\right)$ with a factor $\left(\frac{1}{2 F^{2}}\right) s_{\alpha_{1} \alpha_{3}}$, the term $\left(p_{\beta_{1}}+p_{\beta_{2}}+p_{\beta_{3}}\right)^{2}$ in $\mathcal{U}_{5}^{(3,4)}$ reduces $J\left(\beta_{1}, \beta_{2}, \beta_{3}\right)$ to $J\left(\beta_{1}\right) J\left(\beta_{2}\right) J\left(\beta_{3}\right)$ with a factor $\left(\frac{1}{2 F^{2}}\right) s_{\beta_{1} \beta_{3}}$, while the term $\left(p_{\beta_{2}}+p_{\beta_{3}}+p_{\beta_{4}}\right)^{2}$ 
in $\mathcal{U}_{6}^{(3,4)}$ reduces $J\left(\beta_{2}, \beta_{3}, \beta_{4}\right)$ to $J\left(\beta_{2}\right) J\left(\beta_{3}\right) J\left(\beta_{4}\right)$ with a factor $\left(\frac{1}{2 F^{2}}\right) s_{\beta_{2} \beta_{4}}$. As in the fourpoint example, we can redefine the coefficients so that (4.13) becomes

$$
\begin{aligned}
& \sum_{\sigma \in O P\left(\left\{\alpha_{1}, \alpha_{2}, \alpha_{3}\right\} \bigcup\left\{\beta_{1}, \beta_{2}, \beta_{3}, \beta_{4}\right\}\right)} J(\sigma) \\
= & \mathcal{U}_{1}^{(3,4)} J\left(\alpha_{1}\right) J\left(\alpha_{2}\right) J\left(\alpha_{3}\right) J\left(\beta_{1}\right) J\left(\beta_{2}\right) J\left(\beta_{3}\right) J\left(\beta_{4}\right)+\mathcal{U}_{2}^{\prime(3,4)} J\left(\alpha_{1}\right) J\left(\alpha_{2}\right) J\left(\alpha_{3}\right) J\left(\beta_{1}, \beta_{2}, \beta_{3}\right) J\left(\beta_{4}\right) \\
& +\mathcal{U}_{3}^{\prime(3,4)} J\left(\alpha_{1}\right) J\left(\alpha_{2}\right) J\left(\alpha_{3}\right) J\left(\beta_{1}\right) J\left(\beta_{2}, \beta_{3}, \beta_{4}\right)+\mathcal{U}_{4}^{\prime(3,4)} J\left(\alpha_{1}, \alpha_{2}, \alpha_{3}\right) J\left(\beta_{1}\right) J\left(\beta_{2}\right) J\left(\beta_{3}\right) J\left(\beta_{4}\right) \\
& +\left(\frac{1}{2 F^{2}}\right) J\left(\alpha_{1}, \alpha_{2}, \alpha_{3}\right) J\left(\beta_{1}, \beta_{2}, \beta_{3}\right) J\left(\beta_{4}\right)+\left(\frac{1}{2 F^{2}}\right) J\left(\alpha_{1}, \alpha_{2}, \alpha_{3}\right) J\left(\beta_{1}\right) J\left(\beta_{2}, \beta_{3}, \beta_{4}\right), \quad
\end{aligned}
$$

where

$$
\begin{aligned}
& \mathcal{U}_{2}^{\prime(3,4)}=\mathcal{U}_{2}^{(3,4)}+\left(\frac{1}{2 F^{2}}\right)^{2} a_{1}^{(1,2)} \frac{1}{p_{1}^{2}} s_{\alpha_{1} \alpha_{3}}, \\
& \mathcal{U}_{3}^{\prime(3,4)}=\mathcal{U}_{3}^{(3,4)}+\left(\frac{1}{2 F^{2}}\right)^{2} a_{1}^{(1,2)} \frac{1}{p_{1}^{2}} s_{\alpha_{1} \alpha_{3}}, \\
& \mathcal{U}_{4}^{\prime(3,4)}=\mathcal{U}_{4}^{(3,4)}+\left(\frac{1}{2 F^{2}}\right)^{2} \frac{1}{p_{1}^{2}}\left[b_{1}^{(1,2)} s_{\beta_{1} \beta_{3}}+b_{2}^{(1,2)} s_{\beta_{2} \beta_{4}}\right] .
\end{aligned}
$$

When all the subcurrents go on-shell, the redefined coefficients $\mathcal{U}_{2}^{\prime(3,4)}$ and $\mathcal{U}_{3}^{\prime(3,4)}$ have the same pattern with $\mathcal{V}^{(3,4)}$ (by exchanging the roles of $\alpha$ 's and $\beta$ 's) in the six-point example, while $\mathcal{U}_{4}^{\prime(3,4)}$ has the same pattern with $\mathcal{V}^{(1,5)}$ in the six-point example. For instance, if we consider $\mathcal{U}_{2}^{\prime(3,4)}$

- the coefficients $\mathcal{U}_{2}^{(3,4)}$ get contributions from

- a) the diagrams with the off-shell leg 1 connected to six-point vertices whose other legs are attached to subcurrents containing only $\alpha$ or $\beta$ elements (as shown in figure $1(\mathrm{~B}))$

- b) the diagrams with the off-shell leg 1 connected to four-point vertices, which contain substructures of generalized U(1) identity (as shown in figure 1 (A) ).

Both cases has correspondence in the $\mathcal{U}_{1}^{(2,3)}$ of (4.10) (with exchanging the roles of $\alpha$ 's and $\beta$ 's) and they have the same pattern with (4.10) when the off-shell subcurrents goes on-shell.

- The part $\left(\frac{1}{2 F^{2}}\right)^{2} a_{1}^{(1,2)} \frac{1}{p_{1}^{2}} s_{\alpha_{1} \alpha_{3}}$ is same with $a_{1}^{(1,2)}\left(\frac{1}{2 F^{2}}\right)^{2} \frac{1}{p_{1}^{2}} s_{\beta_{1} \beta_{3}}$ in (4.10) when exchanging the roles of $\alpha$ 's and $\beta$ 's.

Therefore, we can use the off-shell extensions (4.11) and (4.12) corresponding to $\mathcal{V}^{(2,3)}$ and $\mathcal{V}^{(1,4)}$ in the six-point example

$$
\begin{aligned}
& \mathcal{U}_{2}^{\prime(3,4)}=\left(\frac{1}{2 F^{2}}\right)^{2} \frac{1}{p_{1}^{2}}\left[p_{1}^{2}+a_{1}^{(2,3)}\left(p_{\beta_{1}}+p_{\beta_{2}}+p_{\beta_{3}}\right)^{2}\right], \\
& \mathcal{U}_{3}^{\prime(3,4)}=\left(\frac{1}{2 F^{2}}\right)^{2} \frac{1}{p_{1}^{2}}\left[p_{1}^{2}+a_{2}^{(2,3)}\left(p_{\beta_{2}}+p_{\beta_{3}}+p_{\beta_{4}}\right)^{2}\right], \\
& \mathcal{U}_{4}^{\prime(3,4)}=\left(\frac{1}{2 F^{2}}\right)^{2} \frac{1}{p_{1}^{2}} a_{1}^{(1,4)}\left(p_{\alpha_{1}}+p_{\alpha_{2}}+p_{\alpha_{3}}\right)^{2} .
\end{aligned}
$$



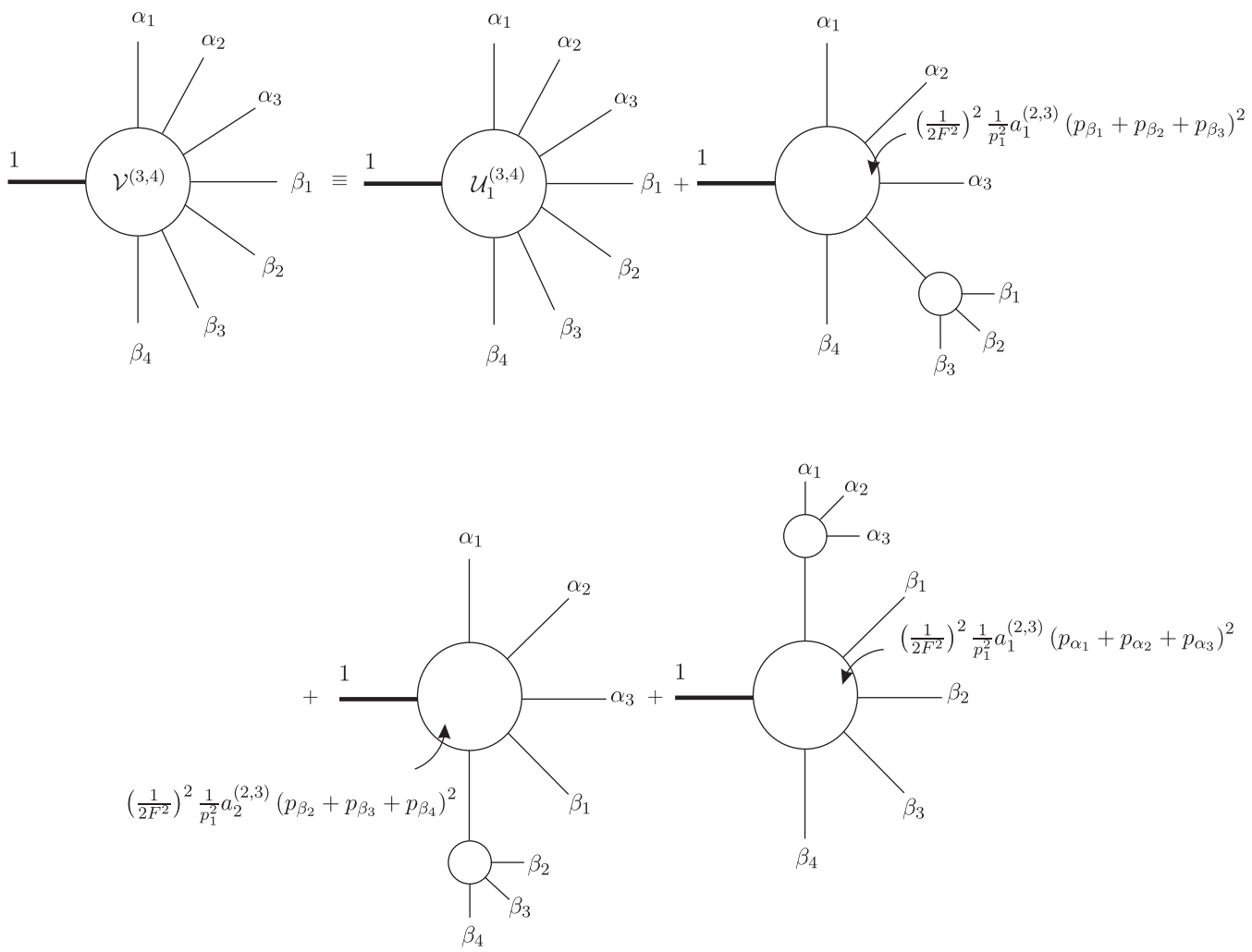

Figure 8. Redefinition of the coefficient of $(3,4)$ division for the identity with three $\alpha$ 's and four $\beta$ 's.

Step-3. Now we notice that $\left(p_{\beta_{1}}+p_{\beta_{2}}+p_{\beta_{3}}\right)^{2}$ in $\mathcal{U}_{2}^{\prime(3,4)}$ reduces $J\left(\beta_{1}, \beta_{2}, \beta_{3}\right)$ to $J\left(\beta_{1}\right) J\left(\beta_{2}\right) J\left(\beta_{3}\right)$ with a factor $\left(\frac{1}{2 F^{2}}\right) s_{\beta_{1} \beta_{3}}$. Thus this term contributes to the $(3,4)$ division. Similarly, the term $\left(p_{\beta_{2}}+p_{\beta_{3}}+p_{\beta_{4}}\right)^{2}$ in $\mathcal{U}_{3}^{\prime(3,4)}$ and $\left(p_{\alpha_{1}}+p_{\alpha_{2}}+p_{\alpha_{3}}\right)^{2}$ in $\mathcal{U}_{4}^{\prime(3,4)}$ reduce $J\left(\beta_{2}, \beta_{3}, \beta_{4}\right)$ and $J\left(\alpha_{1}, \alpha_{2}, \alpha_{3}\right)$ to $J\left(\beta_{2}\right) J\left(\beta_{3}\right) J\left(\beta_{4}\right)$ and $J\left(\alpha_{1}\right) J\left(\alpha_{2}\right) J\left(\alpha_{3}\right)$ respectively. Then, we can rearrange (4.16) again as (figure (8))

$$
\begin{aligned}
& \sum_{\sigma \in O P\left(\left\{\alpha_{1}, \alpha_{2}, \alpha_{3}\right\} \bigcup\left\{\beta_{1}, \beta_{2}, \beta_{3}, \beta_{4}\right\}\right)} J(\sigma) \\
= & \mathcal{V}^{(3,4)} J\left(\alpha_{1}\right) J\left(\alpha_{2}\right) J\left(\alpha_{3}\right) J\left(\beta_{1}\right) J\left(\beta_{2}\right) J\left(\beta_{3}\right) J\left(\beta_{4}\right)+\left(\frac{1}{2 F^{2}}\right)^{2} J\left(\alpha_{1}\right) J\left(\alpha_{2}\right) J\left(\alpha_{3}\right) J\left(\beta_{1}, \beta_{2}, \beta_{3}\right) J\left(\beta_{4}\right) \\
& +\left(\frac{1}{2 F^{2}}\right)^{2} J\left(\alpha_{1}\right) J\left(\alpha_{2}\right) J\left(\alpha_{3}\right) J\left(\beta_{1}\right) J\left(\beta_{2}, \beta_{3}, \beta_{4}\right)+\left(\frac{1}{2 F^{2}}\right)^{2} J\left(\alpha_{1}, \alpha_{2}, \alpha_{3}\right) J\left(\beta_{1}\right) J\left(\beta_{2}\right) J\left(\beta_{3}\right) J\left(\beta_{4}\right) \\
& +\left(\frac{1}{2 F^{2}}\right) J\left(\alpha_{1}, \alpha_{2}, \alpha_{3}\right) J\left(\beta_{1}, \beta_{2}, \beta_{3}\right) J\left(\beta_{4}\right)+\left(\frac{1}{2 F^{2}}\right) J\left(\alpha_{1}, \alpha_{2}, \alpha_{3}\right) J\left(\beta_{1}\right) J\left(\beta_{2}, \beta_{3}, \beta_{4}\right),
\end{aligned}
$$

where

$$
\mathcal{V}^{(3,4)}=\mathcal{U}_{1}^{(3,4)}+\left(\frac{1}{2 F^{2}}\right)^{2} \frac{1}{p_{1}^{2}}\left[a_{1}^{(2,3)} s_{\beta_{1} \beta_{3}}+a_{2}^{(2,3)} s_{\beta_{2} \beta_{4}}+a_{1}^{(1,4)} s_{\alpha_{1} \alpha_{3}}\right]
$$

Thus we only need to prove $\mathcal{V}^{(3,4)}=\left(\frac{1}{2 F^{2}}\right)^{3}$. We leave the proof to the next subsection.

General discussion. In general, when we consider the generalized U(1)-identity (1.1) with $r \alpha$ 's and $s \beta$ 's, we can use Berends-Giele recursion to rewrite the left hand side 
and collect terms corresponding to a same substructure as shown in figure 1. Applying the lower-point identity to the substructures and summing the coefficients for any given division, we reexpress the left hand side of (1.1) by (4.1) or briefly by

$$
\sum_{D} \mathcal{U}_{D}^{(r, s)} J\left(A_{1}\right) \ldots J\left(A_{R_{D}}\right) J\left(B_{1}\right) \ldots J\left(B_{S_{D}}\right)
$$

We start from the divisions with $R_{D}+S_{D}=3$, i.e., $(1,2)$ division and $(2,1)$ division. The contributing diagrams are those in the four-point example with replacing the on-shell lines by off-shell currents. Thus it has the form of the off-shell extension (4.5). Since $p_{A_{i}}^{2} J_{A_{i}}$ and $p_{B_{i}}^{2} J_{B_{i}}$ in (4.5) will further reproduce divisions of $A_{i}$ and $B_{i}$ with coefficients $\sum \frac{1}{p_{1}^{2}} c_{i j} p_{i} \cdot p_{j}$, we absorb all these contributions into the corresponding divisions with $R_{D}+$ $S_{D}>3$. The only left contribution for divisions with $R_{D}+S_{D}=3$ is the first term of (4.5) which gives rise to the expected coefficients.

Then we consider divisions with $R_{D}+S_{D}=5$, which get both contributions from its corresponding $\mathcal{U}_{D}^{(r, s)}$ in (4.21) as well as $p_{A_{i}}^{2} J_{A_{i}}$ and $p_{B_{i}}^{2} J_{B_{i}}$ in the off-shell extension (4.5) of four-point case. Since the coefficients for divisions with $R_{D}+S_{D}=5$ are defined in the same way with the $\mathcal{V}^{\left(r^{\prime}, s^{\prime}\right)}\left(r^{\prime}+s^{\prime}=5\right)$ in the six-point example, they are just the off-shell extensions (4.11), and (4.12). Again, the terms containing $p_{A_{i}}^{2} J_{A_{I}}$ and $p_{B_{i}}^{2} J_{B_{i}}$ in (4.11) and (4.12) are absorbed into the divisions with $R_{D}+S_{D}>5$. The left contributions are those expected coefficients for divisions with $R_{D}+S_{D}=5$.

Redefining the coefficients level by level, we finally have (4.2) where all the coefficients of $R+S<r+s$ divisions match with those in the final formula of the identity (1.1). The coefficient $\mathcal{V}^{(r, s)}$ defined by this method only get contributions from the $\mathcal{U}_{1}^{(r, s)}$ corresponding to the $(r, s)$ division as well as the off-shell extensions of $\mathcal{V}^{(R, S)}$ with $R+S<r+s$. Both cases contain terms proportional to $\frac{1}{p_{1}^{2}} s_{i j}$, where $i$ and $j$ denote arbitrary external on-shell lines. Thus $\mathcal{V}^{(r, s)}$ has the general form

$$
\mathcal{V}^{(r, s)}=\frac{1}{p_{1}^{2}}\left(\sum_{1 \leq i<j \leq r} c_{\alpha_{i} \alpha_{j}} s_{\alpha_{i} \alpha_{j}}+\sum_{1 \leq i<j \leq s} c_{\beta_{i} \beta_{j}} s_{\beta_{i} \beta_{j}}+\sum_{i=1}^{r} \sum_{j=1}^{s} c_{\alpha_{i} \beta_{j}} s_{\alpha_{i} \beta_{j}}\right) .
$$

In the remaining part of this section, we will solve the coefficients $c$ 's to show that $\mathcal{V}^{(r, s)}$ has the expected form.

\subsection{Solving $\mathcal{V}^{(r, s)}$}

In the above discussion, we have shown that the left hand side of the generalized U(1)identity (1.1) could be rearranged into the form (4.2). All the coefficients of divisions in (4.2) with $R_{D}+S_{D}<r+s$ are those on the right hand side of the identity (1.1). Only the coefficient $\mathcal{V}^{(r, s)}$ for $(r, s)$-division are undetermined. Now let us prove that $\mathcal{V}^{(r, s)}$ has the right form, i.e.,

$$
\mathcal{V}^{(r, s)}=\left(\frac{1}{2 F^{2}}\right)^{\frac{r+s-1}{2}} \delta(|r-s|-1)
$$




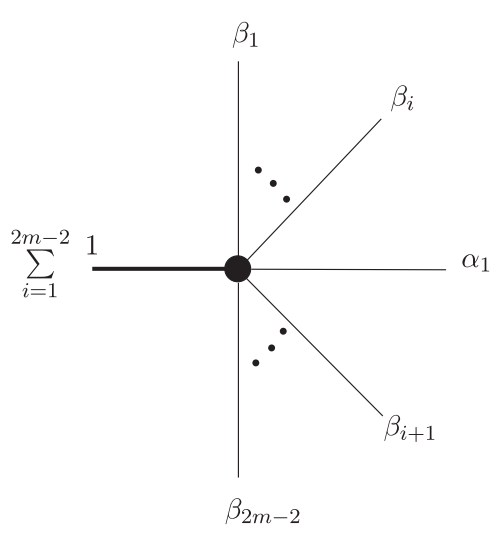

A

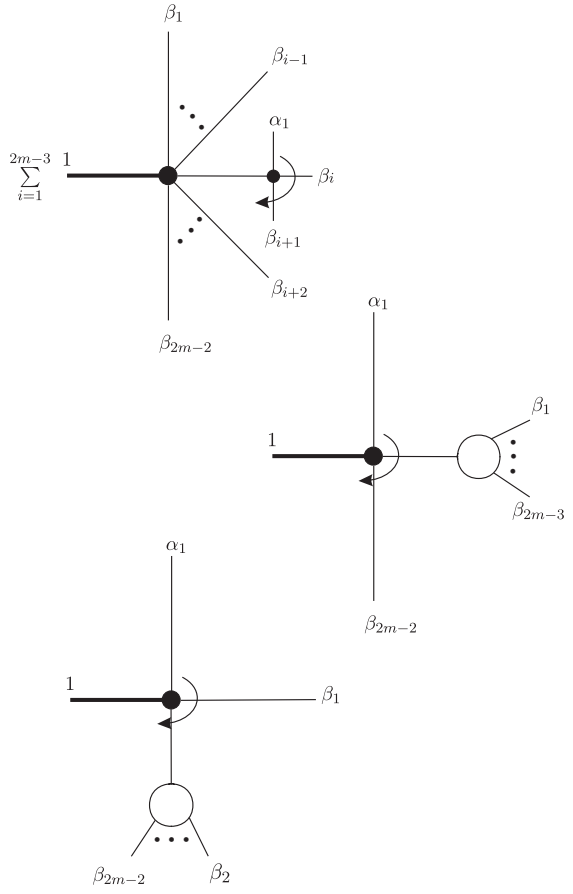

B

Figure 9. Diagrams contributing to $\mathcal{V}^{(1,2 m-2)}$. A curved arrow line denotes the sum over all the possible three positions of $\alpha_{1}$ around the four-point vertices.

\subsection{1 $r=1$}

In this case, (1.1) becomes the $\mathrm{U}(1)$-identity for $2 m=s+2$-point currents, which has been studied in [1]. The $\mathcal{V}^{(1,2)}$ for four-point relation with one $\alpha$ and two $\beta$ 's is

$$
\mathcal{V}^{(1,2)}=\frac{1}{2 F^{2}}
$$

The coefficient $\mathcal{V}^{(1,4)}$ for six-point relation with only one $\alpha$ vanishes. Generically, $\mathcal{V}^{(1,2 m-2)}$ only gets contributions from the diagrams in figure 9 . Following direct calculation which has been shown in [1], we find that

$$
\mathcal{V}^{(1, s)}=0,(\text { for } s>2) \text {. }
$$

Hence $\mathcal{V}^{(1,2 m-2)}$ satisfies the form (4.23).

\subsection{2 $r>1$}

To solve $\mathcal{V}^{(r, s)}$ for $r>1$, we consider the following combination of currents

$$
I\left(\alpha_{1} \mid \alpha_{2}, \ldots, \alpha_{r} ; \beta_{1}, \ldots, \beta_{s}\right) \equiv \sum_{\rho \in O P\left(\left\{\alpha_{2}, \ldots, \alpha_{r}\right\} \bigcup\left\{\beta_{1}, \ldots, \beta_{s}\right\}\right)}\left[\sum_{\sigma \in O P\left(\left\{\alpha_{1}\right\} \bigcup\{\rho\}\right)} J(\sigma)\right],
$$


where we have combined left hand sides of a U(1)-identity and a generalized U(1)identity with $(r-1) \alpha$ 's. Now lets consider the coefficient of the $(r, s)$-division of $I\left(\alpha_{1} \mid \alpha_{2}, \ldots, \alpha_{r} ; \beta_{1}, \ldots, \beta_{s}\right)$. This can be obtained in two different ways:

(a) For a given permutation $\rho \in O P\left(\left\{\alpha_{2}, \ldots, \alpha_{r}\right\} \bigcup\left\{\beta_{1}, \ldots, \beta_{s}\right\}\right)$, we apply the $\mathrm{U}(1)$ identity (2.8) with $\{\rho\}$ as the $\{\beta\}$ set. Then we have

$$
\sum_{\sigma \in O P\left(\alpha_{1} \cup \rho\right)} J(\sigma)=\sum_{\{\rho\} \rightarrow\left\{\rho_{L}\right\}\left\{\rho_{R}\right\}}\left(\frac{1}{2 F^{2}}\right) J\left(\alpha_{1}\right) J\left(\left\{\rho_{L}\right\}\right) J\left(\left\{\rho_{R}\right\}\right) .
$$

Here we summed over divisions $\{\rho\} \rightarrow\left\{\rho_{L}\right\}\left\{\rho_{R}\right\}$ on the right hand side. Substituting above expression into (4.26) and rearranging the summations, we reexpress the combination $I\left(\alpha_{1} \mid \alpha_{2}, \ldots, \alpha_{r} ; \beta_{1}, \ldots, \beta_{s}\right)$ by

$$
\begin{aligned}
& I\left(\alpha_{1} \mid \alpha_{2}, \ldots, \alpha_{r} ; \beta_{1}, \ldots, \beta_{s}\right) \\
& =\sum_{\rho \in O P\left(\left\{\alpha_{2}, \ldots, \alpha_{r}\right\} \bigcup\left\{\beta_{1}, \ldots, \beta_{s}\right\}\right)} \sum_{\{\rho\} \rightarrow\left\{\rho_{L}\right\}\left\{\rho_{R}\right\}}\left(\frac{1}{2 F^{2}}\right) J\left(\alpha_{1}\right) J\left(\left\{\rho_{L}\right\}\right) J\left(\left\{\rho_{R}\right\}\right) \\
& =\sum_{\substack{\left\{\alpha_{2}, \ldots, \alpha_{r}\right\} \rightarrow\left\{\alpha_{L}\right\}\left\{\alpha_{R}\right\} \\
\left\{\beta_{1}, \ldots, \beta_{s}\right\} \rightarrow\left\{\beta_{L}\right\}\left\{\beta_{R}\right\}}}\left(\frac{1}{2 F^{2}}\right) J\left(\alpha_{1}\right)\left[\sum_{\rho_{L} \in O P\left(\left\{\alpha_{L}\right\} \cup\left\{\beta_{L}\right\}\right)} J\left(\left\{\rho_{L}\right\}\right) \sum_{\rho_{R} \in O P\left(\left\{\alpha_{R}\right\} \cup\left\{\beta_{R}\right\}\right)} J\left(\left\{\rho_{R}\right\}\right)\right],
\end{aligned}
$$

where $\sum_{\rho_{L} \in O P\left(\left\{\alpha_{L}\right\} \bigcup\left\{\beta_{L}\right\}\right)} J\left(\left\{\rho_{L}\right\}\right)$ and $\sum_{\rho_{R} \in O P\left(\left\{\alpha_{R}\right\} \cup\left\{\beta_{R}\right\}\right)} J\left(\left\{\rho_{R}\right\}\right)$ are two lower-point substructures of generalized U(1)-identity (2.8). From recursive assumption, we know that both the coefficient $\mathcal{V}^{\left(r_{L}, s_{L}\right)}$ for the $\left(r_{L}, s_{L}\right)$-substructure and the coefficient $\mathcal{V}^{\left(r_{R}, s_{R}\right)}$ for the $\left(r_{R}, s_{R}\right)$-substructure satisfy (4.23). Thus the coefficient $\mathcal{V}_{I}^{(r, s)}$ of the $(r, s)$ division of $I\left(\alpha_{1} \mid \alpha_{2}, \ldots, \alpha_{r} ; \beta_{1}, \ldots, \beta_{s}\right)$ is

$$
\begin{aligned}
\mathcal{V}_{I}^{(r, s)}= & \sum_{\substack{\left\{\alpha_{2}, \ldots, \alpha_{r}\right\} \rightarrow\left\{\alpha_{L}\right\}\left\{\alpha_{R}\right\} \\
\left\{\beta_{1}, \ldots, \beta_{s}\right\} \rightarrow\left\{\beta_{L}\right\}\left\{\beta_{R}\right\}}}\left(\frac{1}{2 F^{2}}\right) \mathcal{V}^{\left(r_{L}, s_{L}\right)} \mathcal{V}^{\left(r_{R}, s_{R}\right)} \\
= & \sum_{\substack{\left\{\alpha_{2}, \ldots, \alpha_{r}\right\} \rightarrow\left\{\alpha_{L}\right\}\left\{\alpha_{R}\right\} \\
\left\{\beta_{1}, \ldots, \beta_{s}\right\} \rightarrow\left\{\beta_{L}\right\}\left\{\beta_{R}\right\}}}\left(\frac{1}{2 F^{2}}\right)^{\frac{r_{L}+s_{L}-1}{2}+\frac{r_{R}+s_{R}-1}{2}+1} \delta\left(\left|r_{L}-s_{L}\right|-1\right) \delta\left(\left|r_{R}-s_{R}\right|-1\right) .
\end{aligned}
$$

The delta functions impose constraints on $r=r_{L}+r_{R}-1$ and $s=s_{L}+s_{R}$. The only nonzero contributions are the cases with $r, s$ satisfying

$$
r=s-1, r=s+1, r=s+3 .
$$

i) For $r=s-1$, only terms with $r_{L}=s_{L}-1$ and $r_{R}=s_{R}-1$ in (4.30) are nonzero. Thus we have

$$
\begin{aligned}
\mathcal{V}_{I}^{(s-1, s)} & =\sum_{s_{L}=1}^{s-1}\left(\frac{1}{2 F^{2}}\right)^{\frac{\left(s_{L}-1\right)+s_{L}-1}{2}+\frac{\left(s-s_{L}-1\right)+\left(s-s_{L}\right)-1}{2}+1} \\
& =\sum_{s_{L}=1}^{s-1}\left(\frac{1}{2 F^{2}}\right)^{s-1}=\left(\frac{1}{2 F^{2}}\right)^{\frac{r+s-1}{2}}(s-1) .
\end{aligned}
$$


ii) For $r=s+1$, both terms with $r_{L}=s_{L}-1, r_{R}=s_{R}+1$ and terms with $r_{L}=s_{L}+1$, $r_{R}=s_{R}-1$ contribute. Then

$$
\begin{aligned}
\mathcal{V}_{I}^{(s+1, s)}= & \sum_{s_{L}=1}^{s-1}\left(\frac{1}{2 F^{2}}\right)^{\frac{\left(s_{L}-1\right)+s_{L}-1}{2}+\frac{\left(s-s_{L}+1\right)+\left(s-s_{L}\right)-1}{2}+1} \\
& +\sum_{s_{L}=0}^{s}\left(\frac{1}{2 F^{2}}\right)^{\frac{\left(s_{L}-1\right)+s_{L}-1}{2}+\frac{\left(s-s_{L}+1\right)+\left(s-s_{L}\right)-1}{2}+1} \\
= & \left(\frac{1}{2 F^{2}}\right)^{\frac{r+s-1}{2}} 2 s .
\end{aligned}
$$

iii) For $r=s+3$, the nonvanishing terms are those with $r_{L}=s_{L}+1, r_{R}=s_{R}+1$. Thus we get

$$
\mathcal{V}_{I}^{(s+3, s)}=\sum_{s_{L}=0}^{s}\left(\frac{1}{2 F^{2}}\right)^{\frac{\left(s_{L}+1\right)+s_{L}-1}{2}+\frac{\left(s-s_{L}+1\right)+\left(s-s_{L}\right)-1}{2}+1}=\left(\frac{1}{2 F^{2}}\right)^{\frac{r+s-1}{2}}(s+1) .
$$

(b) The combination of currents $I\left(\alpha_{1} \mid \alpha_{2}, \ldots, \alpha_{r} ; \beta_{1}, \ldots, \beta_{s}\right)$ can be expressed from another angle: considering a given $\{\rho\} \in O P\left(\left\{\alpha_{1}\right\} \bigcup\left\{\alpha_{2}, \ldots, \alpha_{r}\right\}\right)$ as the $\{\alpha\}$ set on the left hand side of (1.1), we have a combination of currents $\sum_{\{\sigma\} \in O P\left(\{\rho\} \cup\left\{\beta_{1}, \beta_{s}\right\}\right)} J(\sigma)$. After summing over all $\{\rho\} \in O P\left(\left\{\alpha_{1}\right\} \bigcup\left\{\alpha_{2}, \ldots, \alpha_{r}\right\}\right)$, we express $I\left(\alpha_{1}\right.$ । $\left.\alpha_{2}, \ldots, \alpha_{r} ; \beta_{1}, \ldots, \beta_{s}\right)$ by

$$
I\left(\alpha_{1} \mid \alpha_{2}, \ldots, \alpha_{r} ; \beta_{1}, \ldots, \beta_{s}\right)=\sum_{\{\rho\} \in O P\left(\left\{\alpha_{1}\right\} \bigcup\left\{\alpha_{2}, \ldots, \alpha_{r}\right\}\right)}\left[\sum_{\{\sigma\} \in O P\left(\{\rho\} \bigcup\left\{\beta_{1}, \ldots, \beta_{s}\right\}\right)} J(\sigma)\right] .
$$

Expressing each $\sum_{\{\sigma\} \in O P\left(\{\rho\} \bigcup\left\{\beta_{1}, \ldots, \beta_{s}\right\}\right)} J(\sigma)$ by $(4.2)$, we collect the coefficients of $(r, s)$ division for $I\left(\alpha_{1} \mid \alpha_{2}, \ldots, \alpha_{r} ; \beta_{1}, \ldots, \beta_{s}\right)$. There are two parts of contributions $\mathcal{A}^{(r, s)}$ and $\mathcal{B}^{(r, s)}$ :

i) the first part $\mathcal{A}^{(r, s)}$ is the sum of the $\mathcal{V}^{(r, s)}$ coefficients for all possible $\rho \in$ $O P\left(\left\{\alpha_{1}\right\} \bigcup\left\{\alpha_{2}, \ldots, \alpha_{r}\right\}\right)$,

ii) the second part $\mathcal{B}^{(r, s)}$ is the sum of terms with $(r-2, s)$ divisions containing a nontrivial subcurrent $J\left(\phi \in O P\left(\left\{\alpha_{1}\right\} \bigcup\left\{\alpha_{i}, \alpha_{i+1}\right\}\right)\right)$.

As shown in the previous subsection, the terms in $\mathcal{B}^{(r, s)}$ already have the expected coefficients $\left(\frac{1}{2 F^{2}}\right)^{\frac{(r-2)+s-1}{2}} \delta(|r-2-s|-1)$. Collecting the $(r-2, s)$ divisions containing subcurrents $J\left(\alpha_{1}, \alpha_{i}, \alpha_{i+1}\right), J\left(\alpha_{i}, \alpha_{1}, \alpha_{i+1}\right), J\left(\alpha_{i}, \alpha_{i+1}, \alpha_{1}\right)$ and applying the $\mathrm{U}(1)$ identity with one $\alpha$ and two $\beta$ 's, we obtain a term with $(r, s)$ division for $I\left(\alpha_{1} \mid\right.$ $\left.\alpha_{2}, \ldots, \alpha_{r} ; \beta_{1}, \ldots, \beta_{s}\right)$. The coefficient is

$$
\left(\frac{1}{2 F^{2}}\right)^{\frac{(r-2)+s-1}{2}+1} \delta(|r-2-s|-1)=\left(\frac{1}{2 F^{2}}\right)^{\frac{r+s-1}{2}} \delta(|r-2-s|-1) .
$$


After summing over $i=2,3, \ldots, r-1$, we get $\mathcal{B}^{(r, s)}$

$$
\mathcal{B}^{(r, s)}=\sum_{i=2}^{r-1}\left(\frac{1}{2 F^{2}}\right)^{\frac{r+s-1}{2}} \delta(|r-2-s|-1) .
$$

Therefore, $\mathcal{V}_{I}^{(r, s)}$ is given by

$$
\mathcal{V}_{I}^{(r, s)}=\mathcal{A}^{(r, s)}+\mathcal{B}^{(r, s)}=\mathcal{A}^{(r, s)}+\left(\frac{1}{2 F^{2}}\right)^{\frac{r+s-1}{2}}(r-2) \delta(|r-2-s|-1) .
$$

Again, the delta function imposes a constraint on $r$ and $s$. The only nonzero cases are $r=s+1$ and $r=s+3$.

i) For $r=s+1$, we have

$$
\mathcal{V}_{I}^{(s+1, s)}=\mathcal{A}^{(s+1, s)}+\left(\frac{1}{2 F^{2}}\right)^{\frac{r+s-1}{2}}(s-1) .
$$

ii) For $r=s+3$, we have

$$
\mathcal{V}_{I}^{(s+3, s)}=\mathcal{A}^{(s+3, s)}+\left(\frac{1}{2 F^{2}}\right)^{\frac{r+s-1}{2}}(s+1) .
$$

Comparing these expressions of $\mathcal{V}_{I}^{(r, s)}$ derived from (a) approach with those from (b) approach, we immediately conclude that

$$
\mathcal{A}^{(r, s)}=\left(\frac{1}{2 F^{2}}\right)^{\frac{r+s-1}{2}} r \delta(|r-s|-1) .
$$

Then $\mathcal{A}^{(r, s)}$ can be expanded as

$$
\mathcal{A}^{(r, s)}=\left(\frac{1}{2 F^{2}}\right)^{\frac{r+s-1}{2}} \frac{1}{p_{1}^{2}}\left(\sum_{1 \leq i<j \leq r} d_{\alpha_{i} \alpha_{j}} s_{\alpha_{i} \alpha_{j}}+\sum_{1 \leq i<j \leq s} d_{\beta_{i} \beta_{j}} s_{\beta_{i} \beta_{j}}+\sum_{i=1}^{r} \sum_{j=1}^{s} d_{\alpha_{i} \beta_{j}} s_{\alpha_{i} \beta_{j}}\right),
$$

where momentum conservation and on-shell conditions have been used; $d_{i j}(i, j$ can be any $\{\alpha\}$ or $\{\beta\}$ elements) are defined by

$$
d_{\alpha_{i} \alpha_{j}}=d_{\beta_{i} \beta_{j}}=d_{\alpha_{i} \beta_{j}}=r \delta(|r-s|-1) .
$$

If we exchange the roles of $\{\alpha\}$ and $\{\beta\}$ in $I\left(\alpha_{1} \mid \alpha_{2}, \ldots, \alpha_{r} ; \beta_{1}, \ldots, \beta_{s}\right)$, we get another combination of currents

$$
I\left(\alpha_{1}, \ldots, \alpha_{r} ; \beta_{1} \mid \beta_{2}, \ldots, \beta_{s}\right) \equiv \sum_{\rho \in O P\left(\left\{\alpha_{1}, \ldots, \alpha_{r}\right\} \bigcup\left\{\beta_{2}, \ldots, \beta_{s}\right\}\right)}\left[\sum_{\sigma \in O P\left(\{\rho\} \bigcup\left\{\beta_{1}\right\}\right)} J(\sigma)\right],
$$

which has an equivalent form

$$
I\left(\alpha_{1}, \ldots, \alpha_{r} ; \beta_{1} \mid \beta_{2}, \ldots, \beta_{s}\right)=\sum_{\rho \in O P\left(\left\{\alpha_{1}, \ldots, \alpha_{r}\right\} \bigcup\{\rho\}\right)}\left[\sum_{\sigma \in O P\left(\{\rho\} \bigcup\left\{\beta_{1}\right\}\right)} J(\sigma)\right] .
$$



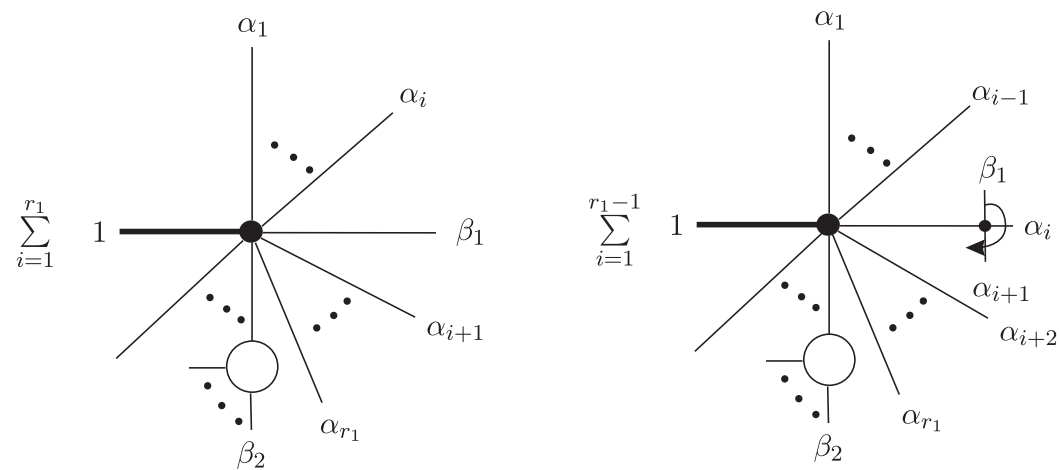

Figure 10. The diagrams cancel out with $\beta_{1}, \beta_{2}$ from reductions of different subcurrents when $r_{1}$ is even.

Following a parallel discussion, we have

$\mathcal{A}^{\prime(s, r)}=\left(\frac{1}{2 F^{2}}\right)^{\frac{r+s-1}{2}} \frac{1}{p_{1}^{2}}\left(\sum_{1 \leq i<j \leq r} d_{\alpha_{i} \alpha_{j}}^{\prime} s_{\alpha_{i} \alpha_{j}}+\sum_{1 \leq i<j \leq s} d_{\beta_{i} \beta_{j}}^{\prime} s_{\beta_{i} \beta_{j}}+\sum_{i=1}^{r} \sum_{j=1}^{s} d_{\alpha_{i} \beta_{j}}^{\prime} s_{\alpha_{i} \beta_{j}}\right)$,

where $\mathcal{A}^{\prime(s, r)}$ is similar with $\mathcal{A}^{(r, s)}$ but defined from $I\left(\alpha_{1}, \ldots, \alpha_{r} ; \beta_{1} \mid \beta_{2}, \ldots, \beta_{s}\right)$ instead. The coefficients $d^{\prime}$ s are given by

$$
d_{\alpha_{i} \alpha_{j}}^{\prime}=d_{\beta_{i} \beta_{j}}^{\prime}=d_{\alpha_{i} \beta_{j}}^{\prime}=s \delta(|s-r|-1)
$$

Recalling that $\mathcal{A}^{(r, s)}$ is given by sum of the $\mathcal{V}^{(r, s)}$ corresponding to different permutations $\{\rho\} \in O P\left(\left\{\alpha_{1}\right\} \bigcup\left\{\alpha_{2}, \ldots, \alpha_{r}\right\}\right)$ in (4.34) and $\mathcal{V}^{(r, s)}$ have the general pattern (4.22), we express $\mathcal{A}^{(r, s)}$ in (4.41) by the general expression (4.22) of $\mathcal{V}^{(r, s)}$. Comparing the coefficients of each $s_{\alpha_{i} \beta_{j}} s_{\alpha_{i} \alpha_{j}}$ and $s_{\beta_{i} \beta_{j}}$ on both sides of (4.41), we obtain a set of equations for $c_{i j}$ where either $i$ or $j$ can be $\alpha$ or $\beta$ elements. Similarly, when expressing $\mathcal{A}^{\prime(s, r)}$ by the $\mathcal{V}^{(r, s)}$ 's corresponding to different permutations $\rho \in O P\left(\left\{\beta_{1}\right\} \bigcup\left\{\beta_{2}, \ldots, \beta_{s}\right\}\right)$ in (4.44), we can also establish the relations between $c_{i j}$ and $d_{i j}^{\prime}$. Let us solve the coefficients $c_{\alpha_{i} \beta_{j}}$, $c_{\alpha_{i} \alpha_{j}}$ and $c_{\beta_{i} \beta_{j}}$ from these equations.

- $c_{\alpha_{i} \beta_{j}}$

We now solve $c_{\alpha_{i} \beta_{j}}$ from their relations with $d_{\alpha_{i} \beta_{j}}$ in (4.41). Noticing that any permutation $\rho$ in the first sum of (4.34) has the general form $\left\{\alpha_{2}, \ldots, \alpha_{i}, \alpha_{1}, \alpha_{i+1}, \ldots, \alpha_{r}\right\}$, the coefficient of $s_{\alpha_{1} \beta_{j}}$ in the second sum should be $c_{\alpha_{i} \beta_{j}}$. This is because the $\alpha_{1}$ in $\left\{\alpha_{2}, \ldots, \alpha_{i}, \alpha_{1}, \alpha_{i+1}, \ldots, \alpha_{r}\right\}$ is inserted at the $i$-th position and plays as the $\alpha_{i}$ in the standard permutation $\left\{\alpha_{1}, \alpha_{2}, \alpha_{3}, \ldots, \alpha_{r}\right\}$. Thus we get the following equation

$$
d_{\alpha_{1} \beta_{j}}=\sum_{i=1}^{r} c_{\alpha_{i} \beta_{j}},(j=1,2, \ldots, s) .
$$




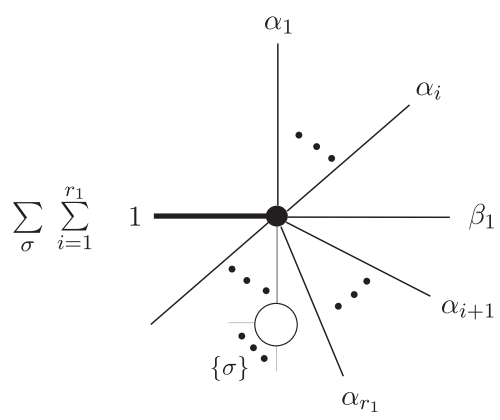

A

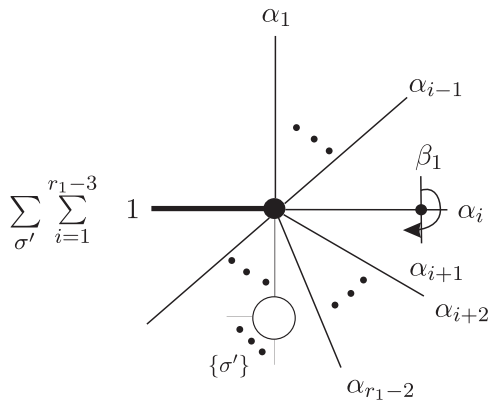

C

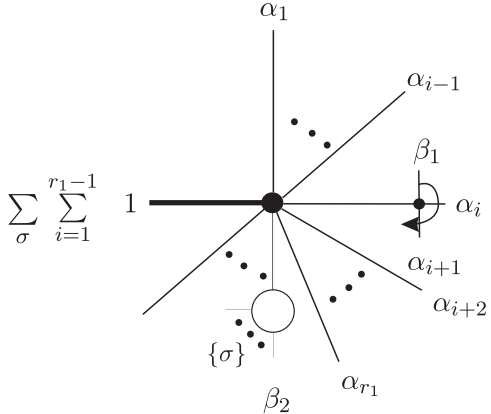

B

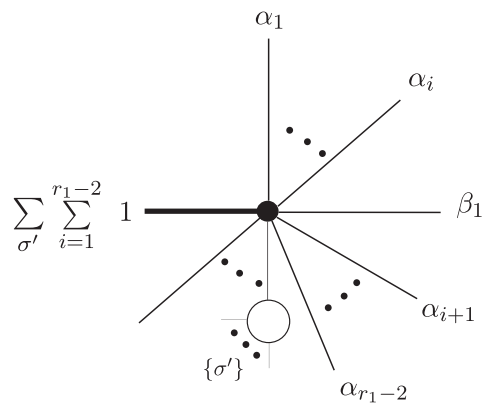

$\mathrm{D}$

Figure 11. The diagrams cancel out with $\beta_{1}, \beta_{2}$ from reductions of different subcurrents when $r_{1}$ is odd. In A and $\mathrm{B}$, we summed over $\sigma \in O P\left(\alpha_{r_{1}+1}, \ldots, \alpha_{r_{1}+s_{1}-1}\right) \bigcup\left\{\beta_{2}, \ldots, \beta_{s_{1}}\right\}$ for given $s_{1}$. In $\mathrm{C}$ and $\mathrm{D}$, we summed over $\sigma^{\prime} \in O P\left(\alpha_{r_{1}-1}, \ldots, \alpha_{r_{1}+s_{1}-1}\right) \bigcup\left\{\beta_{2}, \ldots, \beta_{s_{1}}\right\}$.

Similarly, the coefficient of $s_{\alpha_{l} \beta_{j}}(2 \leq l \leq r)$ in the sum over $\sigma \in$ $O P\left(\left\{\alpha_{2}, \ldots, \alpha_{i}, \alpha_{1}, \alpha_{i+1}, \ldots, \alpha_{r}\right\} \bigcup\{\beta\}\right)$ in (4.34) for given $i$ is

$$
\left\{\begin{array}{c}
c_{\alpha_{l} \beta_{j}} \quad(i<l \leq r) \\
c_{\alpha_{l-1} \beta_{j}}(1<l \leq i-1)
\end{array} .\right.
$$

Thus $d_{\alpha_{i} \beta_{j}}$ with $i=2, \ldots, r$ is given by

$$
d_{\alpha_{i} \beta_{j}}=(l-1) c_{\alpha_{l} \beta_{j}}+(r-l+1) c_{\alpha_{l-1} \beta_{j}},(l=2, \ldots, r, j=1 \ldots s) .
$$

In the same way, when considering $\mathcal{A}^{\prime(s, r)}$ and the combination (4.43), we obtain the relations between $d^{\prime}$ 's and $c$ 's

$$
\begin{aligned}
& d_{\alpha_{i} \beta_{1}}^{\prime}=\sum_{j=1}^{s} c_{\alpha_{i} \beta_{j}},(i=1,2, \ldots, r) \\
& d_{\alpha_{i} \beta_{j}}^{\prime}=(k-1) c_{\alpha_{i} \beta_{k}}+(s-k+1) c_{\alpha_{i} \beta_{k-1}},(i=1, \ldots, r, k=2 \ldots s) .
\end{aligned}
$$

We first prove that $c_{\alpha_{1} \beta_{1}}=c_{\alpha_{1} \beta_{2}}$. Considering positions of $\beta_{1}$ and $\beta_{2}$, we can classify the contributing diagrams into two types: 
i) $\beta_{1}$ and $\beta_{2}$ come from reduction of a same subcurrent.

ii) $\beta_{1}$ and $\beta_{2}$ come from reduction of different subcurrents.

The factors $c_{\alpha_{1} \beta_{1}}$ and $c_{\alpha_{1} \beta_{2}}$ receive equal contributions from the first types of diagrams. For the second type, we can always find diagrams cancel with each other. To see this, we assume that the last $\alpha$ element in front of $\beta_{2}$ (or in the same substructure with $\beta$ ) is $\alpha_{r_{1}}$.

If $r_{1}$ is even, the diagrams are typically given by figure 10 . The left diagram in figure 10 contribute a $\frac{r_{1}}{2} s_{\alpha_{1} \beta_{1}},{ }^{5}$ while the right diagram contribute a $-\frac{r_{1}}{2} s_{\alpha_{1} \beta_{1}}$. Thus these two contributions to $c_{\alpha_{1} \alpha_{1}}$ cancel out. Since the $r_{1}$ is even, there are odd number of legs in front of $\beta_{2}$. From Feynman rules, such diagrams do not contribute to $c_{\alpha_{1} \beta_{2}}$.

If $r_{1}$ is odd, the diagrams in figure 11 should be taken into account. The A, B diagrams of figure 11 contribute $\frac{r_{1}-2}{2}$ and $-\frac{r_{1}-2}{2}$ to $c_{\alpha_{1} \beta_{1}}$, while the diagrams $\mathrm{C}$ and $\mathrm{D}$ of figure 11 contribute $\frac{r_{1}-4}{2}$ and $-\frac{r_{1}-4}{2}$ to $c_{\alpha_{1} \beta_{1}}$. Thus $c_{\alpha_{1} \beta_{1}}$ does not get any nonzero contribution from figure 11 . When considering $c_{\alpha_{1} \beta_{2}}$, we find that diagrams A, B, C, D in figure 11 contribute $r_{1},-\left(r_{1}-1\right), r_{1}-3,-\left(r_{1}-2\right)$. Thus $c_{\alpha_{1} \beta_{2}}$ also does not get any nonzero contribution from these diagrams.

Now we substitute $c_{\alpha_{1} \beta_{1}}=c_{\alpha_{1} \beta_{2}}$ into (4.51) with $i=1, k=2$ and remember $d^{\prime}$ have the form (4.46) we have

$$
c_{\alpha_{1} \beta_{1}}=c_{\alpha_{1} \beta_{2}}=\delta(|r-s|-1) .
$$

Inserting $c_{\alpha_{1} \beta_{2}}$ into (4.51) with $i=1, k=3$, we get

$$
c_{\alpha_{1} \beta_{3}}=\delta(|r-s|-1),
$$

Inserting $c_{\alpha_{1} \beta_{3}}$ into (4.51) with $i=1, k=4$, we get

$$
c_{\alpha_{1} \beta_{4}}=\delta(|r-s|-1) .
$$

Repeating these steps, we can obtain $c_{\alpha_{1} \beta_{k}}=\delta(|r-s|-1)$ from $d_{\alpha_{1} \beta_{k}}^{\prime}$ where $k=$ $2, \ldots, s$.

We then substitute $c_{\alpha_{1} \beta_{1}}$ into (4.49) with $l=2, j=1$. Recalling that $d$ have the form (4.42), we get

$$
c_{\alpha_{2} \beta_{1}}=\delta(|r-s|-1) .
$$

Substituting $c_{\alpha_{2} \beta_{1}}$ into (4.51) with $i=2, k=2$, we get

$$
c_{\alpha_{2} \beta_{2}}=\delta(|r-s|-1) .
$$

\footnotetext{
${ }^{5}$ For convenience, we neglect a factor $\left(\frac{1}{2 F^{2}}\right)^{\frac{r+s-1}{2}}$ in the remaining discussion and put the factor back in the final result.
} 
Substituting $c_{\alpha_{2} \beta_{2}}$ into (4.51) with $i=2, k=3$, we get

$$
c_{\alpha_{2} \beta_{3}}=\delta(|r-s|-1) .
$$

Repeating these steps, we solve that $c_{\alpha_{2} \beta_{k}}=\delta(|r-s|-1)$ from $d_{\alpha_{2} \beta_{k}}^{\prime}$ where $k=$ $2, \ldots, s$.

Following similar discussions and considering all the equations (4.49) and (4.51), we finally solve all the coefficients

$$
c_{\alpha_{i} \beta_{j}}=\delta(|r-s|-1),(i=1, \ldots, r, j=1, \ldots, s) .
$$

- $c_{\alpha_{i} \alpha_{j}}$ and $c_{\beta_{i} \beta_{j}}$.

We consider $d_{\alpha_{i} \alpha_{j}}^{\prime}$ in (4.45). $d_{\alpha_{i} \alpha_{j}}^{\prime}$ gets a $c_{\alpha_{i} \alpha_{j}}$ from each $\rho \in O P\left(\left\{\beta_{1}\right\} \bigcup\left\{\beta_{2}, \ldots, \beta_{s}\right\}\right)$. Thus we arrive at

$$
s c_{\alpha_{i} \alpha_{j}}=s \delta(|s-r|-1) .
$$

Then $c_{\alpha_{i} \alpha_{j}}$ are solved as

$$
c_{\alpha_{i} \alpha_{j}}=\delta(|s-r|-1) .
$$

If we consider $d_{\alpha_{i} \alpha_{j}}$ instead, we can solve $c_{\beta_{i} \beta_{j}}$ from (4.41) in the same way. The solution is

$$
c_{\beta_{i} \beta_{j}}=\delta(|s-r|-1)
$$

To sum up, all the coefficients $c_{\alpha_{i} \alpha_{j}}, c_{\beta_{i} \beta_{j}}$ and $c_{\alpha_{i} \beta_{j}}$ in (4.22) have the form $\delta(|s-r|-1)$. Considering on-shell condition and momentum conservation, the sum in (4.22) then give rise

$$
\mathcal{V}^{(r, s)}=\left(\frac{1}{2 F^{2}}\right)^{\frac{r+s-1}{2}} \frac{1}{p_{1}^{2}} p_{1}^{2} \delta(|s-r|-1)=\left(\frac{1}{2 F^{2}}\right)^{\frac{r+s-1}{2}} \delta(|s-r|-1) .
$$

\section{Conclusions}

In this paper, we proposed and proved the generalized U(1)-identity for tree-level off-shell currents in nonlinear sigma model. When we take on-shell limit, this relation becomes the on-shell generalized U(1) identity which is equivalent with KK relation. The U(1)identity for off-shell currents proposed in [1] is a special case of the generalized U(1)-identity. There are several possible further extensions of this work, including the generalized off-shell BCJ relation, the loop-level extensions and the BCJ duality which implies the relations in nonlinear sigma model.

\section{Acknowledgments}

Y. J. Du would like to acknowledge the EU programme Erasmus Mundus Action 2, Project 9 and the International Postdoctoral Exchange Fellowship Program of China for supporting his postdoctoral research in Lund University (with Fudan University as the home university). Y. J. Du's research is supported in parts by the NSF of China Grant 
No.11105118, China Postdoctoral Science Foundation No.2013M530175 and the Fundamental Research Funds for the Central Universities of Fudan University No.20520133169. G. Chen, S. Y. Li and H. Q. Liu's research has been supported by the Fundamental Research Funds for the Central Universities under contract 020414340080, NSF of China Grant under contract 11405084, the Jiangsu Ministry of Science and Technology under contract BK20131264 and by the Swedish Research Links programme of the Swedish Research Council (Vetenskapsradets generella villkor) under contract 348-2008-6049. We also thank Baoyi Chen, Edna Cheung, Yunxuan Li, Ruofei Xie, Yuan Xin for useful discussion.

Open Access. This article is distributed under the terms of the Creative Commons Attribution License (CC-BY 4.0), which permits any use, distribution and reproduction in any medium, provided the original author(s) and source are credited.

\section{References}

[1] G. Chen and Y.-J. Du, Amplitude relations in non-linear $\sigma$-model, JHEP 01 (2014) 061 [arXiv: 1311.1133] [INSPIRE].

[2] Z. Bern, J.J.M. Carrasco and H. Johansson, New relations for gauge-theory amplitudes, Phys. Rev. D 78 (2008) 085011 [arXiv: 0805.3993] [inSPIRE].

[3] R. Kleiss and H. Kuijf, Multi-gluon cross-sections and five jet production at hadron colliders, Nucl. Phys. B 312 (1989) 616 [INSPIRE].

[4] N.E.J. Bjerrum-Bohr, P.H. Damgaard and P. Vanhove, Minimal basis for gauge theory amplitudes, Phys. Rev. Lett. 103 (2009) 161602 [arXiv:0907.1425] [INSPIRE].

[5] S. Stieberger, Open \& closed vs. pure open string disk amplitudes, arXiv:0907.2211 [INSPIRE].

[6] V. Del Duca, L.J. Dixon and F. Maltoni, New color decompositions for gauge amplitudes at tree and loop level, Nucl. Phys. B 571 (2000) 51 [hep-ph/9910563] [INSPIRE].

[7] B. Feng, R. Huang and Y. Jia, Gauge amplitude identities by on-shell recursion relation in S-matrix program, Phys. Lett. B 695 (2011) 350 [arXiv:1004.3417] [INSPIRE].

[8] H. Tye and Y. Zhang, Remarks on the identities of gluon tree amplitudes, Phys. Rev. D 82 (2010) 087702 [arXiv: 1007.0597] [inSPIRE].

[9] Y.-X. Chen, Y.-J. Du and B. Feng, A proof of the explicit minimal-basis expansion of tree amplitudes in gauge field theory, JHEP 02 (2011) 112 [arXiv:1101.0009] [INSPIRE].

[10] F. Cachazo, Fundamental BCJ relation in $N=4 S Y M$ from the connected formulation, arXiv: 1206.5970 [INSPIRE].

[11] Z. Bern, T. Dennen, Y.-T. Huang and M. Kiermaier, Gravity as the square of gauge theory, Phys. Rev. D 82 (2010) 065003 [arXiv: 1004.0693] [INSPIRE].

[12] N.E.J. Bjerrum-Bohr, P.H. Damgaard, H. Johansson and T. Sondergaard, Monodromy-like relations for finite loop amplitudes, JHEP 05 (2011) 039 [arXiv:1103.6190] [INSPIRE].

[13] J.J. Carrasco and H. Johansson, Five-point amplitudes in $N=4$ super-Yang-Mills theory and $N=8$ supergravity, Phys. Rev. D 85 (2012) 025006 [arXiv:1106.4711] [INSPIRE].

[14] R.H. Boels and R.S. Isermann, New relations for scattering amplitudes in Yang-Mills theory at loop level, Phys. Rev. D 85 (2012) 021701 [arXiv:1109.5888] [INSPIRE]. 
[15] R.H. Boels and R.S. Isermann, Yang-Mills amplitude relations at loop level from non-adjacent BCFW shifts, JHEP 03 (2012) 051 [arXiv: 1110.4462] [INSPIRE].

[16] Z. Bern, J.J.M. Carrasco, L.J. Dixon, H. Johansson and R. Roiban, Simplifying multiloop integrands and ultraviolet divergences of gauge theory and gravity amplitudes, Phys. Rev. D 85 (2012) 105014 [arXiv:1201.5366] [INSPIRE].

[17] J.J.M. Carrasco, M. Chiodaroli, M. Günaydin and R. Roiban, One-loop four-point amplitudes in pure and matter-coupled $N \leq 4$ supergravity, JHEP 03 (2013) 056 [arXiv:1212.1146] [INSPIRE].

[18] Y.-J. Du and H. Lüo, On general BCJ relation at one-loop level in Yang-Mills theory, JHEP 01 (2013) 129 [arXiv:1207.4549] [INSPIRE].

[19] S. Oxburgh and C.D. White, BCJ duality and the double copy in the soft limit, JHEP 02 (2013) 127 [arXiv: 1210.1110] [INSPIRE].

[20] R. Saotome and R. Akhoury, Relationship between gravity and gauge scattering in the high energy limit, JHEP 01 (2013) 123 [arXiv:1210.8111] [INSPIRE].

[21] R.H. Boels, B.A. Kniehl, O.V. Tarasov and G. Yang, Color-kinematic duality for form factors, JHEP 02 (2013) 063 [arXiv:1211.7028] [INSPIRE].

[22] R.H. Boels, R.S. Isermann, R. Monteiro and D. O'Connell, Colour-kinematics duality for one-loop rational amplitudes, JHEP 04 (2013) 107 [arXiv:1301.4165] [INSPIRE].

[23] N.E.J. Bjerrum-Bohr, T. Dennen, R. Monteiro and D. O'Connell, Integrand oxidation and one-loop colour-dual numerators in $N=4$ gauge theory, JHEP 07 (2013) 092 [arXiv: 1303.2913] [INSPIRE].

[24] Z. Bern, S. Davies, T. Dennen, Y.-T. Huang and J. Nohle, Color-kinematics duality for pure Yang-Mills and gravity at one and two loops, arXiv:1303.6605 [INSPIRE].

[25] J. Nohle, Color-kinematics duality in one-loop four-gluon amplitudes with matter, Phys. Rev. D 90 (2014) 025020 [arXiv: 1309.7416] [INSPIRE].

[26] C.R. Mafra, O. Schlotterer and S. Stieberger, Explicit BCJ numerators from pure spinors, JHEP 07 (2011) 092 [arXiv: 1104.5224] [INSPIRE].

[27] R. Monteiro and D. O'Connell, The kinematic algebra from the self-dual sector, JHEP 07 (2011) 007 [arXiv: 1105.2565] [INSPIRE].

[28] N.E.J. Bjerrum-Bohr, P.H. Damgaard, R. Monteiro and D. O'Connell, Algebras for amplitudes, JHEP 06 (2012) 061 [arXiv:1203.0944] [INSPIRE].

[29] C.-H. Fu, Y.-J. Du and B. Feng, An algebraic approach to BCJ numerators, JHEP 03 (2013) 050 [arXiv: 1212.6168] [INSPIRE].

[30] R. Monteiro and D. O'Connell, The kinematic algebras from the scattering equations, JHEP 03 (2014) 110 [arXiv:1311.1151] [INSPIRE].

[31] J. Broedel and J.J.M. Carrasco, Virtuous trees at five and six points for Yang-Mills and gravity, Phys. Rev. D 84 (2011) 085009 [arXiv:1107.4802] [INSPIRE].

[32] C.-H. Fu, Y.-J. Du and B. Feng, Note on symmetric BCJ numerator, JHEP 08 (2014) 098 [arXiv: 1403.6262] [INSPIRE].

[33] S.G. Naculich, Scattering equations and virtuous kinematic numerators and dual-trace functions, JHEP 07 (2014) 143 [arXiv: 1404.7141] [INSPIRE]. 
[34] F. Cachazo, S. He and E.Y. Yuan, Scattering equations and Kawai-Lewellen-Tye orthogonality, Phys. Rev. D 90 (2014) 065001 [arXiv:1306.6575] [INSPIRE].

[35] F. Cachazo, S. He and E.Y. Yuan, Scattering of massless particles in arbitrary dimensions, Phys. Rev. Lett. 113 (2014) 171601 [arXiv:1307.2199] [INSPIRE].

[36] F. Cachazo, S. He and E.Y. Yuan, Scattering of massless particles: scalars, gluons and gravitons, JHEP 07 (2014) 033 [arXiv:1309.0885] [INSPIRE].

[37] S.G. Naculich, Scattering equations and BCJ relations for gauge and gravitational amplitudes with massive scalar particles, JHEP 09 (2014) 029 [arXiv:1407.7836] [INSPIRE].

[38] Z. Bern and T. Dennen, A color dual form for gauge-theory amplitudes, Phys. Rev. Lett. 107 (2011) 081601 [arXiv:1103.0312] [INSPIRE].

[39] Y.-J. Du, B. Feng and C.-H. Fu, The construction of dual-trace factor in Yang-Mills theory, JHEP 07 (2013) 057 [arXiv: 1304.2978] [INSPIRE].

[40] C.-H. Fu, Y.-J. Du and B. Feng, Note on construction of dual-trace factor in Yang-Mills theory, JHEP 10 (2013) 069 [arXiv:1305.2996] [INSPIRE].

[41] Y.-J. Du, B. Feng and C.-H. Fu, Dual-color decompositions at one-loop level in Yang-Mills theory, JHEP 06 (2014) 157 [arXiv: 1402.6805] [INSPIRE].

[42] T. Bargheer, S. He and T. McLoughlin, New relations for three-dimensional supersymmetric scattering amplitudes, Phys. Rev. Lett. 108 (2012) 231601 [arXiv:1203.0562] [INSPIRE].

[43] Q. Ma, Y.-J. Du and Y.-X. Chen, On primary relations at tree-level in string theory and field theory, JHEP 02 (2012) 061 [arXiv:1109.0685] [INSPIRE].

[44] Y.-J. Du, B. Feng and C.-H. Fu, BCJ relation of color scalar theory and KLT relation of gauge theory, JHEP 08 (2011) 129 [arXiv:1105.3503] [INSPIRE].

[45] K. Kampf, J. Novotny and J. Trnka, Recursion relations for tree-level amplitudes in the $\mathrm{SU}(N)$ nonlinear $\sigma$-model, Phys. Rev. D 87 (2013) 081701 [arXiv:1212.5224] [INSPIRE].

[46] K. Kampf, J. Novotny and J. Trnka, Tree-level amplitudes in the nonlinear $\sigma$-model, JHEP 05 (2013) 032 [arXiv: 1304.3048] [INSPIRE].

[47] F.A. Berends and W.T. Giele, Multiple soft gluon radiation in parton processes, Nucl. Phys. B 313 (1989) 595 [inSPIRE]. 